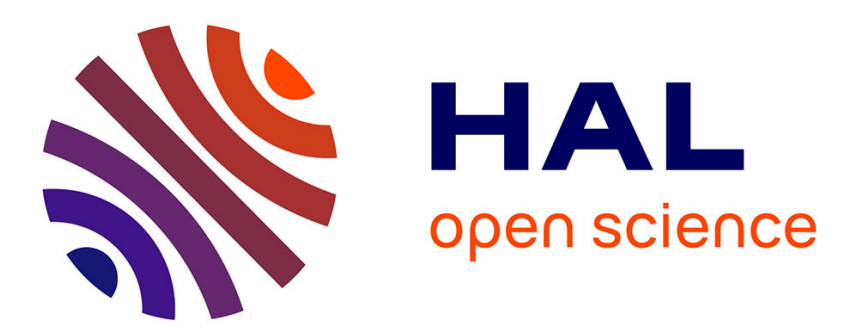

\title{
Knowledge representation and qualitative simulation of salmon redd functioning. Part II : Qualitative model of redds
}

Francois Guerrin, Jacques Dumas

\section{- To cite this version:}

Francois Guerrin, Jacques Dumas. Knowledge representation and qualitative simulation of salmon redd functioning. Part II: Qualitative model of redds. BioSystems, 2001, 59 (2), pp.85-108. 10.1016/s0303-2647(01)00101-0 . hal-02681325

\section{HAL Id: hal-02681325 \\ https://hal.inrae.fr/hal-02681325}

Submitted on 31 May 2020

HAL is a multi-disciplinary open access archive for the deposit and dissemination of scientific research documents, whether they are published or not. The documents may come from teaching and research institutions in France or abroad, or from public or private research centers.
L'archive ouverte pluridisciplinaire HAL, est destinée au dépôt et à la diffusion de documents scientifiques de niveau recherche, publiés ou non, émanant des établissements d'enseignement et de recherche français ou étrangers, des laboratoires publics ou privés. 


\title{
Knowledge representation and qualitative simulation of salmon redd functioning. Part II: qualitative model of redds
}

\author{
F. Guerrin ${ }^{\mathrm{a}, *}$, J. Dumas ${ }^{\mathrm{b}}$ \\ a Inra, Unité de Biométrie et Intelligence artificielle, BP 27, 31326 Castanet-Tolosan Cedex, France \\ ${ }^{\mathrm{b}}$ Inra, Station d'Hydrobiologie, BP 3, 64310 St-Pée-sur-Nivelle, France
}

Received 21 December 1999; received in revised form 03 October 2000; accepted 23 October 2000

\begin{abstract}
This paper describes a qualitative model of the functioning of salmon redds (spawning areas of salmon) and its impact on mortality rates of early stages. For this, we use $Q$ sim, a qualitative simulator, which appeared adequate for representing available qualitative knowledge of freshwater ecology experts (see Part I of this paper). Since the number of relevant variables was relatively large, it appeared necessary to decompose the model into two parts, corresponding to processes occurring at separate time-scales. A qualitative clock allows us to submit the simulation of salmon developmental stages to the calculation of accumulated daily temperatures (degree-days), according to the clock ticks and a water temperature regime set by the user. Therefore, this introduces some way of real-time dating and duration in a purely qualitative model. Simulating both sub-models, either separately or by means of alternate transitions, allows us to generate the evolutions of variables of interest, such as the mortality rates according to two factors (flow of oxygenated water and plugging of gravel interstices near the bed surface), under various scenarios. (C) 2001 Elsevier Science Ireland Ltd. All rights reserved.
\end{abstract}

Keywords: Qualitative simulation; Dynamical systems; Knowledge representation; Time scales; Salmon redd ecology

\section{Introduction}

For piedmont streams such as the Nivelle River (Basque country, France), the survival rate at the egg and alevin stages beneath the streambed surface has been shown critical for salmon, as it controls its population dynamics. This was emphasized by a stochastic model of the salmon

\footnotetext{
* Corresponding author. Present address: Cirad, BP 20, 97408 Saint-Denis, Reunion Island, France..

E-mail address: guerrin@cirad.fr (F. Guerrin).
}

life-cycle called CBS (Dumas et al., 1996; Faivre et al., 1997). Spawning of salmon occurs in December in upstream reaches. Spawned eggs develop according to the following sequence of stages: eyed-embryos appear in January, hatching occurs in February, and emergence of fry occurs during March or early April, when the yolk sac resorption is completed (Dumas and Darolles, 1999). Redds are built by females at the upstream limit of riffles with an area of a few square meters. They are made of a pit followed, in the stream axis, by a gravel and cobble dome under which 
egg pockets are located at $15-25 \mathrm{~cm}$ depth beneath the surface (Hobbs, 1937; White, 1942; Stuart, 1953; Chapman, 1988). When digging a redd, females remove a large proportion of fine particles (silt and sand) off the substrate (McNeil and Ahnell, 1964; Peterson and Quinn, 1996). Thus they increase both the gravel porosity and permeability favoring the flow of oxygenated water within the redd to eggs. The shape of a redd (a dome) also favors the intergravel water flow. During the intragravel life of salmon, the water discharge drives the changes in spawning gravel by means of scour and fill processes and, therefore, impacts the survival of eggs and alevins through two main mortality factors: oxygen depletion and clogging of superficial gravel layer by fines. Actually, fine-sediment infiltration as well as redd planing off by stormflow events are responsible for low survival rates. Filling up the interstices in spawning gravels, fine particles reduce the intergravel flow velocity and, consequently, decrease the amount of dissolved oxygen supplied to eggs and alevins. Even, plugging near-surface interstices by fines may block the emergence of fry (Harris, 1978; Chapman, 1988; Lisle and Lewis, 1992; Crisp, 1993).

Understanding how redd functioning may impact the mortality of fish at early developmental stages (egg, embryo, alevin, fry) is of great concern to fish ecologists. Exploring the feasibility of modeling and simulating the functioning of redds is thus a challenging activity. With this aim, we must emphasize the lack of valid and usable mathematical models due to fluid mechanics complexity in such fractured porous media (existence of fissures, non-constant porosity, etc.), which parameters are actually unknown. Larger-scale quantitative mathematical models, e.g. linking watershed characteristics to trout habitat and population dynamics (Jessup, 1998) or relating flow and temperature regimes to individual length and abundance of trout (van Winkle et al., 1998), do exist. However, due to the great imprecision of field data and uncertainty on salmonid ecology, simulation outputs of such quantitative models do not provide the user with much more than qualitative assessments although uncertainty can be dealt with by introducing stochastic parameters in the models (like in van Winkle et al., 1998). Therefore, the interest of a quantitative approach is very weak (this is further discussed in Section $6)$.

Fortunately, there exists an empirical knowledge coming from field measurements and observations made by freshwater ecologists that can be found in the international literature (like in Chapman, 1988). Knowledge often comes from correlations made on field data from which only qualitative features may be exploited (e.g. 'Generally there exists a decreasing relation between the amount of fine particles in the redd and the overall survival rate'). No precise type of such functions may be derived from the data, as in practice they widely vary according to time, location, hydrological regime, fish species, etc. (Lisle and Lewis, 1992). Quantifying the involved phenomena could only be achieved on heavily instrumented and controlled experimental facilities. In fact, for the problem we want to address, one has at hand only partial qualitative information or incomplete and imprecise quantitative data which, in the case of the Nivelle river, are relatively coarse (Dumas et al., 1998). As a consequence, it is very difficult to use a numerical modeling approach. Therefore, our goal is to study to what extent qualitative modeling and simulation can help us in making use of expert informal knowledge to perform simulations.

As a first step, a naive model (i.e. mainly based on common sense knowledge) was implemented with aim to assess the adequacy of Qsim (the qualitative simulator that was chosen; see Part I) to represent available knowledge and provide us with sound simulations. Then, few meetings with freshwater ecologists allowed us to formalize a conceptual model summarizing the relationships holding among relevant variables. A preliminary version of a Qsim model was quickly implemented, which was then refined in further versions. This first version was an aggregate model composed of more than 30 variables. It presented the major problem of bringing together variables corresponding, actually, to different time-scales:

- a slower scale (week), corresponding to the biological processes of fish development and related mortality; 
- a faster scale (some hours or 1 day), corresponding to the physical processes of fine-sediment infiltration and redd plugging under stormflow events.

The simulation was hence governed by the fastest scale, richer in event occurrences, which led the simulation to branch into numerous irrelevant distinct behaviors from a single initial state. To solve that problem, the current version was made by partitioning this model into two connected submodels via transitions on shared variables: developmental stage of fish, dissolved oxygen, and fine particles infiltration. This decomposition was performed by hand (the causal structure was already known), not automatically as described by Clancy and Kuipers (1997). A preliminary version of this work was presented in Guerrin et al. (1997).

In Part I of this paper, we have provided unfamiliar readers with the bases of the Qsim formalism. A simple example taken from the salmon redd problem (sensitivity of fish to dissolved oxygen depletion) was given to illustrate the Qualitative Differential Equation (QDE) ontology and simulation. Here we describe the structure and give simulation results of the QDEs accounting for:

- a qualitative autonomous clock, the clock$Q D E$, allowing for accumulation of degreedays from average water temperatures (Section 2);

- the slower scale processes (i.e. fish development), modeled as a slow-QDE, making the fish grow in age according to Celsius degreedays accrued by the clock, and die according to dissolved oxygen concentration and fines accumulation (Section 3);

- the faster scale processes (i.e. fine sediment infiltration and redd clogging), modeled as a fast-QDE (Section 4).

Whereas the slow-QDE involves only informal biological knowledge, the fast-QDE provides us, in addition, with an opportunity to illustrate the use of Qsim in making use of partial analytical knowledge by abstracting ordinary differential equations (ODEs) into a qualitative differential equation (QDE) - rain-flow relation modeled as a linear reservoir Section 4.1.
Coupling both slow and fast models by means of qualitative transitions is presented in Section 5. Finally, discussion of this work and further perspectives are in Section 6.

\section{Qualitative autonomous clock (clock-QDE)}

\subsection{Clock-QDE structure}

It is known that changes in salmon development, i.e. passing from one stage to another, depend upon the moving sum of average daily water temperatures, expressed quantitatively as Celsius degree-days. Stage changes are determined from spawning (0 degree-days), according to the following thresholds derived from our ecological knowledge:

- eyed-embryo: $\approx 250$ degree-days,

- hatching: $\approx 450$ degree-days,

- beginning of emergence: $\approx 900$ degree-days,

- end of emergence: $\approx 1050$ degree-days.

The clock-QDE, borrowed from Ben Kuipers's (1994) book (pp. 228-229), based on QDE transitions, generates clock discontinuous ticks and their count over time; here, each tick is assumed to represent a 10 days period. This QDE was modified by addition of two variables (see Fig. 1 and complete code in Appendix A):

- temp, standing for a regime of average (quantitative) temperatures for each 10 days period, computed as a list of integers stored into a global variable set by the user like: $(0.10)(n-$ $0.10)(n-1.5), \ldots$, where $n-0, n-1$, etc. are landmarks created during the simulation in Ndate's qspace (variable accounting for ticks count; its qspace was initially set to (0 inf)), and integers after the dot are the water temperature average values (for sake of simplicity, values are rounded up to the nearest $5^{\circ} \mathrm{C}$ multiple);

- Ndaydeg, the degree-days accrued, calculated as the moving sum of temperatures holding successively for each 10 days period (from the temp variable).

Two specific Lisp functions (Lisp fctn1 in Fig. 1) have been written to: (i) pop elements up of the list of temperatures at each transition state created during the simulation (i.e. at each clock tick); 


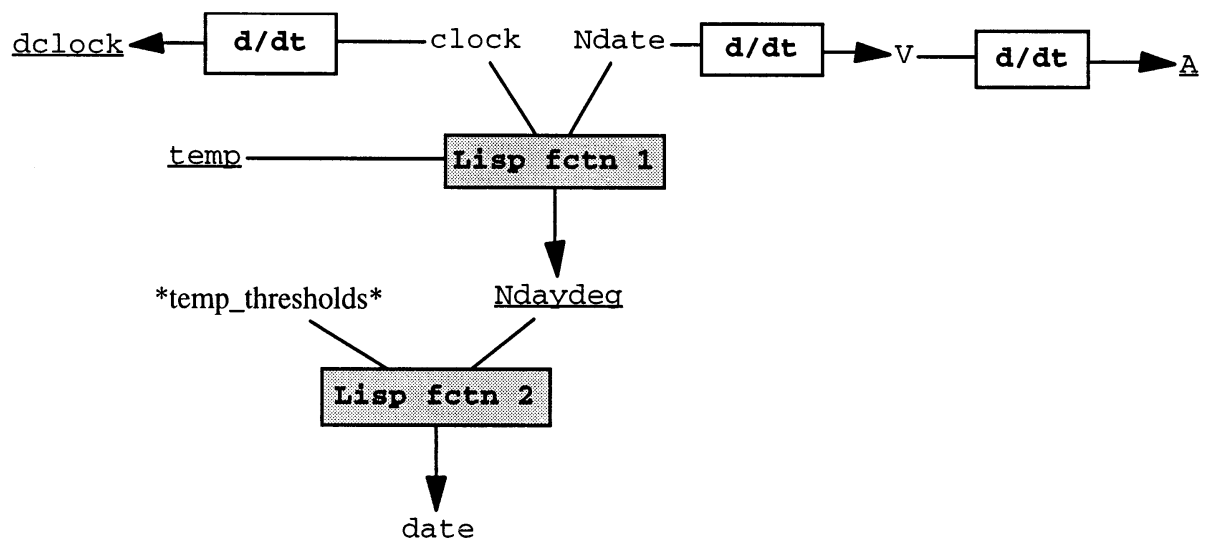

Fig. 1. Graphical representation of the clock-QDE. $x_{1}, \ldots, x_{n} \rightarrow y$ denotes $y=f\left(x_{1}, \ldots, x_{n}\right)$ with $x_{1}, \ldots, x_{n}, y$ the variables and $f$ the constraint (in white boxes); variables with constant constraints are underlined. In grey boxes are special Lisp functions that do not belong to the QDE as well as the Lisp variable *temp_thresholds* (see text); date is defined in the slow-QDE (Section 3).

and (ii) sum them up in order to build dynamically Ndaydeg quantity space - initialized in the define-qde clause as (0 inf).

\subsection{Clock-QDE simulation}

Simulating the clock motion generates a single behavior (Fig. 2) exhibiting, in addition to the ticks sequence (clock) and their count (Ndate), the representation of the temperature regime (temp) and the degree-days accumulation (Ndaydeg) see Fig. 3. Once performed the clock simulation, constructing an alist with both Ndate and Ndaydeg quantity spaces (which include new landmarks created by Qsim - see the $Y$ axes of these variables plots on Fig. 3) and comparing it with the critical degree-days thresholds (listed above and represented by the Lisp variable *temp_thresholds* as shown Fig. 1) allow us to extract the sub-list of critical dates at which changes occur. They are landmarks of date, variable defined in the slow-QDE (as we will see Section 3). This is performed by Lisp fctn2 appearing in Fig. 1.

This was made as an attempt to introduce some notion of temporal dating and duration in such purely qualitative models. Assuming that each clock tick represents a 10 days period, each new created landmark of Ndate (which denotes ticks count) gives a date, expressed as the number of 10 days periods elapsed since the initial state at $T_{0}$. For example (see Fig. 3), at $T_{7}$ the $n-6$ landmark reached by Ndate denotes, actually, that 70 days have passed (the end of the first 10 days period is labelled $n-0)$. At that time, temperature (temp) jumped from $5^{\circ} \mathrm{C}$ in the preceding period to $10^{\circ} \mathrm{C}$ at the current one, while the number of degreedays accrued (Ndaydeg), that added up to 400 before, discretely jumps to 450 .

Then, comparing the thresholds determining changes in stage to the degree-days accrued (Ndaydeg qmags), allows us to generate the critical dates for stage transitions. They are: $0, n-2, n-6, n-10, \quad$ and $n-11$, denoting the $0,30,70,110,120$ th day since spawning. They correspond to the following stages: spawning, eyed-embryo, hatching, beginning and end of emergence, respectively. For purposes of clarity, correspondences between simulation time $T_{\mathrm{i}}$, Ndate landmarks, realistic approximate dates, and stage transitions, are summarized in Table 1.

Occurrence of stage transitions are presented on Fig. 4, according to two slightly different temperature regimes: temp (upper part of Fig. 4) and temp2 (lower part of Fig. 4). Stage versus date phase-space representation provides us with a means to compare dating: note on Fig. 4 that temp2 results in a 10 days advance in stage occurrences compared to temp. 
Fig. 2. Behavior tree of the clock-QDE, exhibiting a single behavior white dots with vertical bar denote transition states created at each clock tick.

\section{Qualitative model of fish development $($ slow-QDE)}

\subsection{Slow-QDE structure}

For sake of clarity, all the variables and quantity spaces of the slow-QDE are summarized in Table 2; its graphical representation, showing the relationships linking its variables through the constraints, is in Fig. 5, and complete code is given in Appendix B.

Starting from the QDE1 example described in Part I, where we already defined three variables (stage, dstage, Sens-O2) and their constraints, we first need to complete the model by introducing the variable date. Its qspace is an output of the clock-QDE simulation (see Fig. 1), given as a list of critical dates where transition from one stage to another occurs, according to degree-days accumulation (see Table 2). As date denotes the critical dates for stage transitions, both variables are positively monotonically related. Thus, an $M+$ constraint, with one-to-one corresponding values, holds between these variables (see graphical representation in Fig. 6).

Mortality due to any specific factor corresponds to the integration over time of $L(t)=S(t) \times P(t)$ (for $L$, intensity of lethal factor; $S$, sensitivity to factor; $P$, factor pressure) sort of generalization of the Ohm's law $\left(I=1 / R_{\Omega} \times U\right)$. Two mortality factors are taken into account: amount of dissolved oxygen available to fish (described in the fastQDE Section 4) and accumulation of fine sediments on the bed surface that can make a sort of 'physical barrier' to fry emergence. They correspond to the following variables (see qspaces in Table 2):

- Sens-O2 and Sens-barr, accounting for sensitivities of fish to lethal factors;

- $P$-O2 and $P$-barr, pressures of lethal factors;

- $L$-O2 and $L$-bar, intensities of lethal factors.

We already set the $H$ - constraint holding among stage and Sens-O2 (see Part I). The rela- tion between stage and Sens-barr is given as an $S+$ constraint, assuming that the sensitivity of fish to the physical barrier only holds after alevins are about to emerge from the redd (i.e. when $q m a g($ stage $\left.) \geq e m_{1}\right)$, then it increases up to a higher value $(\mathrm{sb})$ reached at the end of emergence, and stays steady beyond (see Fig. 7).

Straightforwardly, the products $L(t)=S(t) \times$ $P(t)$ for both mortality factors are given by two mult constraints:

((mult $P$-O2 Sens-O2 L-O2)),

((mult Sens-barr $P$-barr $L$-barr)).

Intensities of lethal factors $L(t)$ are kinds of lethality (or toxicity) flows, assumed to be proportional to mortality rates: $\mu(t)=k L(t)$. Assuming constant $k>0$, this is equivalent to $\mu(t)=\mathrm{M}_{0}^{+}$ $(L(t)),{ }^{1}$ that can be approximated as $\mu(t) \approx L(t)$. Therefore, $L-\mathrm{O} 2$ and $L$-barr may be directly interpreted qualitatively as mortality rates for each of the factors oxygen and accumulation of fines on the redd. Their sum, $L$-tot, thus represents the total mortality rate dependent upon these cause:

((add $L$-O2 $L$-barr $L$-tot)).

\subsection{Slow-QDE simulation}

In order to simulate the slow-QDE one must define an initial state. For example, let us start from the following specification, to be put in the assert-values slot of the slow-QDE simulation function (like in the QDE1 example in Part I):

(stage (0 nil))

(dstage ((0 inf) nil))

$(P-\mathrm{O} 2$ (pb nil))

$(P$-barr $((0 \mathrm{pb})$ nil $))$.

\footnotetext{
${ }^{1}$ Short-hand notation for an $\mathrm{M}^{+}$constraint linking $L(t)$ to $R(t)$ with $(00)$ corresponding values.
} 

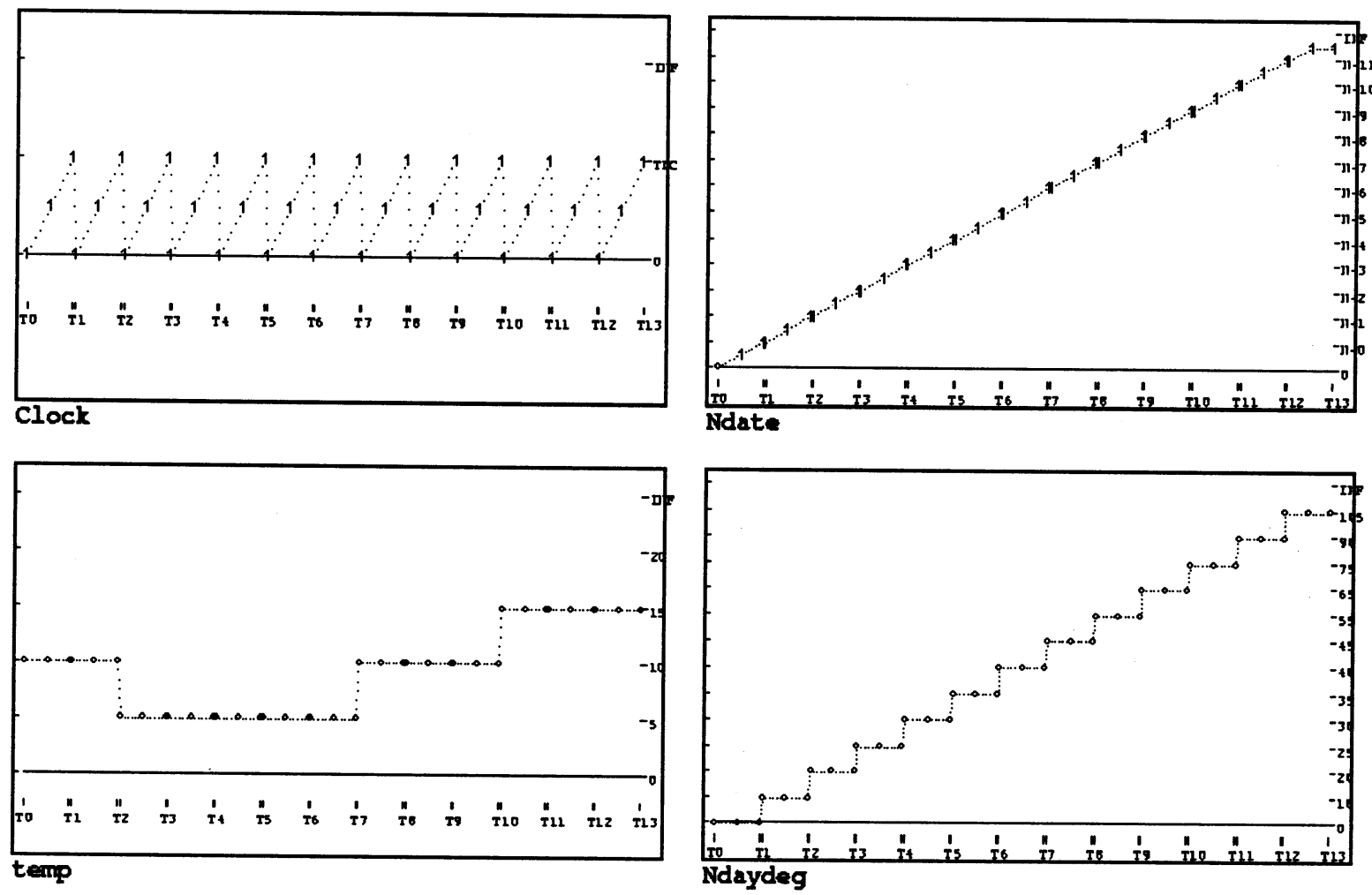

Fig. 3. Qualitative plots of the clock-QDE simulated behavior. Clock denotes the clock motion as ticks succession (transition states $T_{1}, T_{2}, T_{3}, \ldots$ ), Ndate the ticks count (expressed as $n-0, n-1, \ldots$, assumed to be 10 days periods), temp a water temperature regime, and Ndaydeg the amount of degree-days accumulated over time $(\times 10)$.

It specifies that we start with stage at spawning, dstage $>0, P-\mathrm{O} 2$ at its higher additional landmark, $P$-barr below its non-null landmark - i.e. in the closed interval $(0 \mathrm{pb})$. Note that, although the same symbol $\mathrm{pb}$ is given to landmarks of two different qspaces, they are taken as different by Qsim since, as outlined in Part I, landmark definition is local to a given qspace. Here we assume $P$-O2 and $P$-barr are constant (constant constraints are added to the slow-QDE - see Appendix B).

We get three behaviors, branching on the same time interval (Fig. 8): behavior 1 with 13 states, behaviors 2 and 3 with 11 states each. Behavior 1 is on Fig. 9, where most interesting variables only are plotted. Stage increases continuously as expected. $L-\mathrm{O} 2$ exhibits the same kind of qualitative behavior as Sens-O2 in QDE1 (see Part I). How- ever, $L$-barr remains 0 from $T_{0}$ to $T_{4}$, then increases right after $T_{4}$ (when emergence begins) up to a new landmark dynamically created at $T_{6}$ where simulation ends (transition rule is fired when stage is increasing at em2). Therefore, $L$-tot exhibits a more complex behavior, summarizing these two variables contributions: $L-\mathrm{O} 2$ alone until $T_{4}$, both variables beyond. $L$-tot reaches a first maximum at $T_{3}$ (due to $L-\mathrm{O} 2$ peak at hatching), then a local minimum at $T_{4}$ (as $L-\mathrm{O} 2$ decreases after hatching and $L$-barr is still zero until alevins emergence), then a new maximum at $T_{6}$ (due to $L$-barr additional contribution). Based on $L$-tot, comparing the three behaviors, shows no difference until $T_{4}$ (for behavior 1 see Fig. 9, for behaviors 2 and 3 see Fig. 10). Differences come after, according to the qualitative magnitude reached by $L$-tot at the end of the simulation: 
either it is above its first maximum at $T_{3}$ (behavior 1), or below (behavior 2), or the same (behavior 3). Qsim, here, determines three possible behaviors, according to the respective ordering of two events concerning the dynamics of both stage and $L$-tot:

- $L$-tot crosses its $L$-3 landmark (at $T_{5}$ ) before stage has reached em2 (at $\left.T_{6}\right)$ : this gives behavior 1 ;

- stage reaches em2 (at $T_{5}$ ) while $L$-tot is still below $L-3$ when simulation ends: this gives behavior 2 ;

- $L$-tot reaches $L-3$ and stage reaches em 2 at the same time $\left(T_{5}\right)$ : this gives behavior 3 .

This phenomenon, called occurrence branching, appears because information is lacking in the model about the genuine ordering between both events: $L$-tot at $L-3$, and stage at em2. Although this is often undesirable when it results in irrelevant distinctions among behaviors (solutions exist to eliminate it - see Fouché (1992)), we believe, here, that all these three behaviors are equally possible, according to the respective magnitudes of $L-\mathrm{O} 2$ and $L$-barr. Therefore, behaviors 1,2 , and 3 can be all considered genuine.

Table 1

Correspondences between simulation time, Ndate landmarks, realistic approximate dates, and stage transitions, in the clock simulation presented Fig. 3.

\begin{tabular}{llll}
\hline Time & Ndate & Actual dates & Stage \\
\hline $\mathrm{T}_{0}$ & 0 & December, 10 & 0 \\
$\mathrm{~T}_{1}$ & $n-0$ & December, 20 & - \\
$\mathrm{T}_{2}$ & $n-1$ & January, 1 & - \\
$\mathrm{T}_{3}$ & $n-2$ & January, 10 & eye \\
$\mathrm{T}_{4}$ & $n-3$ & January, 20 & - \\
$\mathrm{T}_{5}$ & $n-4$ & February, 1 & - \\
$\mathrm{T}_{6}$ & $n-5$ & February, 10 & - \\
$\mathrm{T}_{7}$ & $n-6$ & February, 20 & hat \\
$\mathrm{T}_{8}$ & $n-7$ & March, 1 & - \\
$\mathrm{T}_{9}$ & $n-8$ & March, 10 & - \\
$\mathrm{T}_{10}$ & $n-9$ & March, 20 & em1 \\
$\mathrm{T}_{11}$ & $n-10$ & April, 1 & em2 \\
$\mathrm{T}_{12}$ & $n-11$ & April, 10 & - \\
\hline
\end{tabular}

\section{Qualitative model of redd filling by fines ( fast $Q D E)$}

\subsection{Fast-QDE structure}

Similarly to the slow-QDE structure, all the variables and quantity spaces of the fast-QDE are summarized in Table 3; a graphical representation of the QDE is in Fig. 11, and complete code is given in Appendix C.

We consider here the process of redd filling by fines occurring at a 1 day scale. Basically, it is dependent upon the streamflow. First we introduce a simple rain-flow relation, modeling a catchment as a linear reservoir (as described in Haan et al., 1982). Storage $V(t)$ is directly proportional to the outflow $Q(t)$ (flow brought by the catchment to the river): $V(t)=k Q(t)$, for $k>0$, a proportionality constant (storage coefficient). The difference between inflow (here the rain, $R(t)$ ) and outflow $Q(t)$ is the time rate of change of the storage: $\mathrm{d} v / \mathrm{d} t=R(t)-Q(t)$, or by substitution, $k(\mathrm{~d} Q / \mathrm{d} t)=R(t)-Q(t)$, or $(\mathrm{d} Q / \mathrm{d} t)+(Q(t) / k)=$ $R(t) / k$, the solution for which is: $Q(t)=R(t) \times$ $\left(1-\mathrm{e}^{(-\tau / k)}\right)$. Since $k>0$, we have the qualitative approximation:

$\frac{\mathrm{d} Q}{\mathrm{~d} t} \approx R(t)-Q(t)$.

In order to 'abstract' the above ordinary differential equation into a QDE, we define three variables (see Table 3): rain, $Q$ (flow excess brought to the river with two additional landmarks accounting for critical values influencing both the total suspended solids increase and the dome height decrease) and $\mathrm{d} Q$ (with a qspace assuming this derivative may be positive, negative or null). Eq. (5) is then abstracted by two constraints:

$((\mathrm{d} / \mathrm{d} t Q \mathrm{~d} Q))$,

((add $\mathrm{d} Q Q$ rain)).

In order to get the time rate of change of rain, we can differentiate Eq. (5). It comes:

$\frac{\mathrm{d}^{2} Q}{\mathrm{~d} t^{2}} \approx \frac{\mathrm{d} R}{\mathrm{~d} t}-\frac{\mathrm{d} Q}{\mathrm{~d} t}$. 

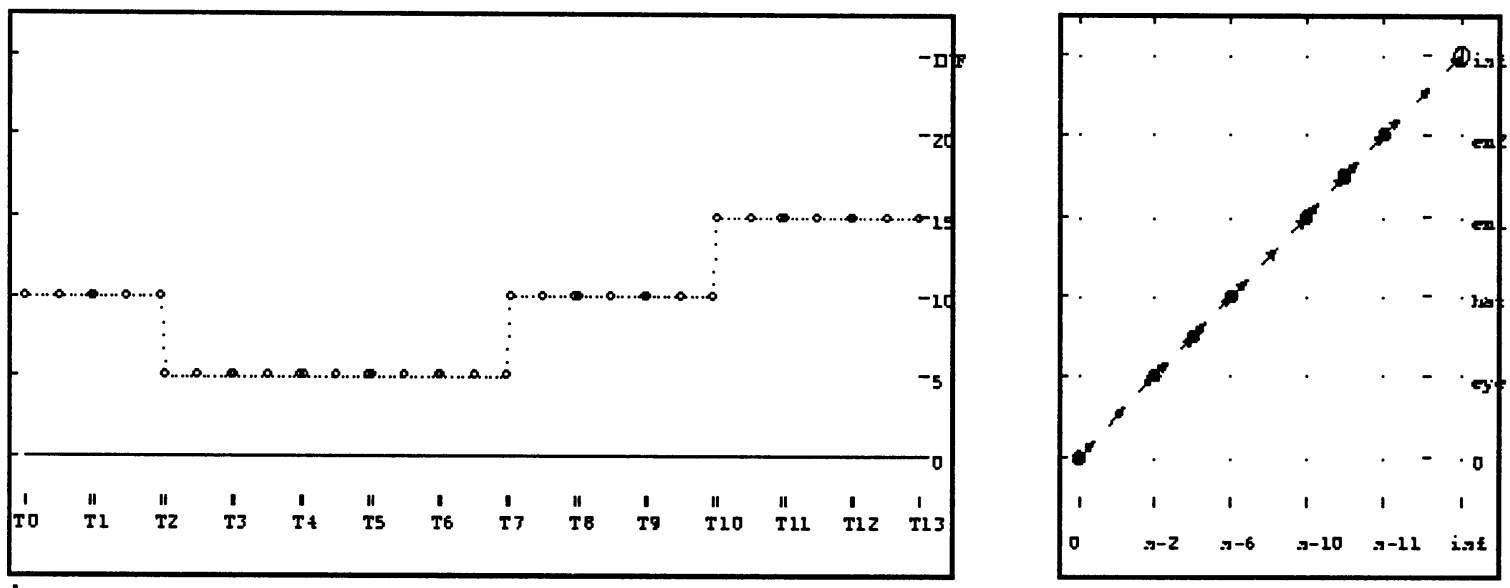

temp
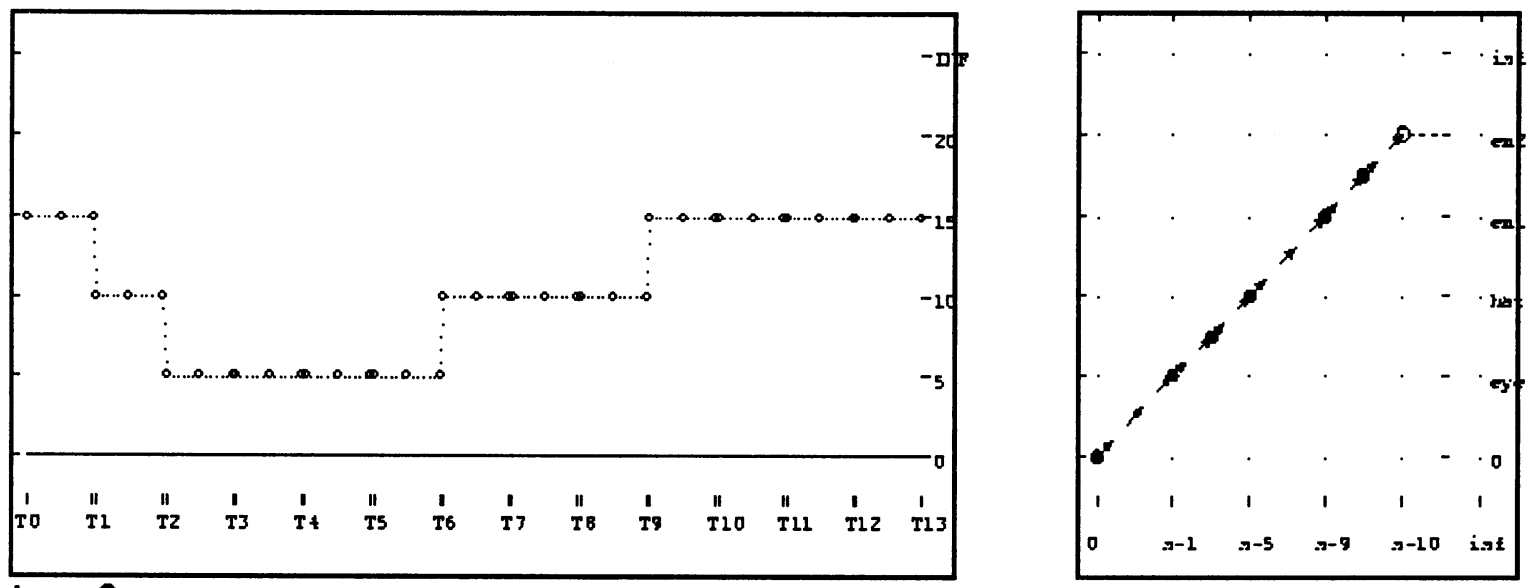

temp2

Fig. 4. An example of qualitative dating: stage ( $Y$ axis) vs. date ( $X$ axis) phase-space representation (on the handside); date denotes critical dates (as 10 days periods, $n-2, n-6, \ldots$ ) at which stage changes according to water average temperature regimes (temp and temp2). Note that temp2 induces an advance of 10 days in stage evolution compared to temp.

Defining $\mathrm{dd} Q$ and drain variables with default qspaces, Eq. (8) is then formed by three constraints:

$((\mathrm{d} / \mathrm{d} t \mathrm{~d} Q \mathrm{dd} Q))$

$((\mathrm{d} / \mathrm{d} t$ rain drain) $)$,

((add $\mathrm{dd} Q \mathrm{~d} Q$ drain)).

Moreover, we specify dd $Q$ as a constant, that is for all $t$ its qdir is set to 0. Due to the add constraint (expression 11), this guarantees that $\mathrm{d} Q$ and drain have the same qdir.
Two factors influencing the process of fine-sediment accumulation are considered: the amount of total suspended solids (TSS) carried out by the river, and the height of the dome of gravel constituting the redd. TSS and dome are defined with positive additional landmarks. Both are influenced by the excess of the streamflow $Q$. These influences are, respectively positive and negative. As such, they are modeled as increasing or decreasing saturation curves using $S+$ and $S$ - constraints, respectively (see Figs. 12 and 13) with following meanings: 
Table 2

Slow-QDE: qualitative variables and quantity spaces

\begin{tabular}{lll}
\hline Variables & Quantity spaces & Comments \\
\hline date & $\begin{array}{l}(0 n-2 n-6 n-10 \\
n-11 \mathrm{inf})\end{array}$ & $\begin{array}{l}\text { Critical dates at which stage transitions occur as generated by the clock-QDE (see Section 2); } \\
\text { landmarks match with stage landmarks }\end{array}$ \\
stage & $\begin{array}{l}(0 \text { eye hat em1 } \\
\text { em2 inf })\end{array}$ & $\begin{array}{l}\text { Development stage of fish. 0: spawning; eye: eyed-embryo; hat: hatching em1, em2: beginning } \\
\text { and end of emergence } \\
\text { stage rate of change }\end{array}$ \\
dstage & $(0$ inf $)$ & Sensitivity of fish to oxygen depletion. lo: low; md: medium; hi: high \\
Sens-O2 & $(0$ lo md hi inf $)$ & Pressure of lethal factor oxygen. pa: low critical value; pb: high critical value \\
$P$-O2 & $(0$ pa pb inf $)$ & Intensity of lethal factor oxygen \\
$L$-O2 & $(0$ inf $)$ & Sensitivity of fish to fines deposition. 0: no sensitivity; sb: critical value \\
Sens-barr & $(0 \mathrm{sb}$ inf $)$ & Pressure of lethal factor fines deposition 0: normal value; pb: critical value \\
$P$-barr & $(0 \mathrm{pb}$ inf $)$ & Intensity of lethal factor fines deposition \\
$L$-barr & $(0 \mathrm{inf})$ & Total intensity of lethal factors $L$-O2 and $L$-barr \\
$L$-tot & $(0$ inf $)$ &
\end{tabular}

- when a minimal value qa of flow $Q$ is reached, ${ }^{2}$ TSS increases from its corresponding value sa and dome decreases from its corresponding value $d$;

- When $Q$ reaches some higher value qb, TSS reaches a new corresponding value $\mathrm{sb}>\mathrm{sa}$, and dome falls down to 0 ; both remain constant beyond (i.e. for $q \operatorname{mag}(Q)>\mathrm{qb}$ ).

The more TSS there are in the water, the faster the fines accumulation, and conversely. Defining by fines the amount of sediment infiltrated within the redd and by dfine its time derivative, we thus state an $\mathrm{M}+$ monotonic constraint between TSS and dfine (Fig. 14). Corresponding values in the constraint state that when TSS tends to 0 , dfine tends to $-\infty$ (accumulation speed is infinitely decreasing) and there exist two critical values of TSS: sa for which dfine is 0 (fines are steady), sb for which dfine is df; when TSS tends to infinity so does dfine (accumulation speed is infinitely increasing).

According to Lotspeich and Everest (1981) the inner structure of a redd can be characterized by two quantities:

- The geometric mean particle diameter of gravels:

\footnotetext{
${ }^{2}$ For sake of simplicity we assume that the same minimal value qa starts the influence of $Q$ on both variables TSS and dome.
}

$$
\mathrm{dg}=\prod_{i=0}^{n} d_{i}^{\omega_{i}}
$$

with $d_{i}$ the median diameter and $\omega_{i}$ the weight percentage of particles retained on sieve $i$;

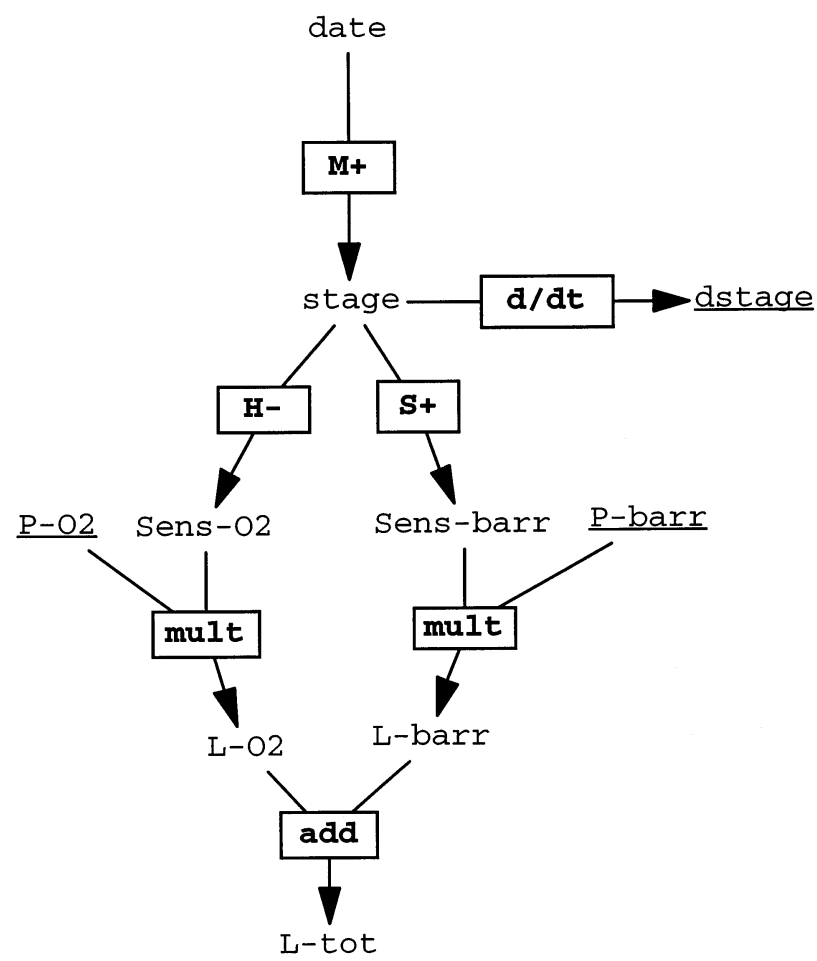

Fig. 5. Graphical representation of the slow-QDE (same legend as in Fig. 1). 


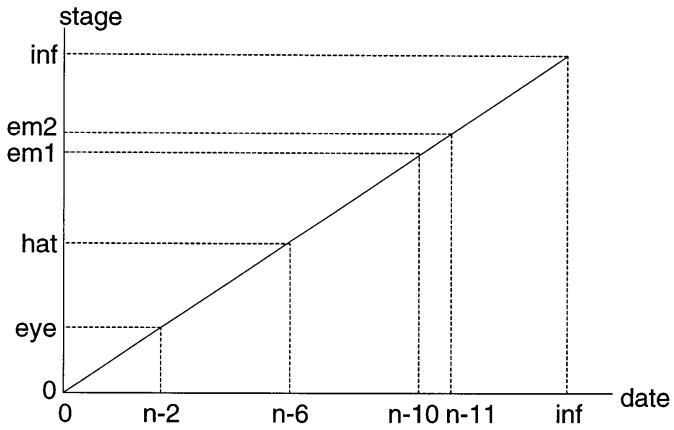

Fig. 6. Graphical representation of the relationship holding between stage and date modeled as the $\mathrm{M}+$ constraint: $((\mathrm{M}+$ date stage $)(00)(n-2$ eye $)(n-6$ hat $)(n-10$ eml $)(n-$ $11 \mathrm{em} 2)$ (inf inf)).

- an heterogeneity coefficient: $S_{0}=\left(d_{75} / d_{25}\right)^{1 / 2}$, with $d_{25}$ and $d_{75}$ the particle size of the 25th and 75 th percentiles of the weight of a sample. Whereas $\mathrm{dg}>0$, we have $S_{0}>1$ ( 1 stands for maximal homogeneity). Thus, dg and $S_{0}$ are assigned qspaces with additional landmarks as shown in Table 3. The amount of fines influences negatively $\mathrm{dg}$ and positively $S_{0}$ : mean diameter decreases and heterogeneity increases as long as fines increase up to a limit value $\mathrm{fp}$. These relations are modeled as $S-$ and $S+$ constraints, respectively (Figs. 15 and 16).

The fredle index, developed by Lotspeich and Everest (1981) as the ratio $F_{i}=\left(\mathrm{dg} / S_{0}\right)$, synthesizes both infuences of the mean size of the substrate and particle size dispersion. It gives a good indication on the substrate permeability (Chapman, 1988). $F_{i}$, dg and $S_{0}$ are related by a mult constraint with two sets of corresponding values:

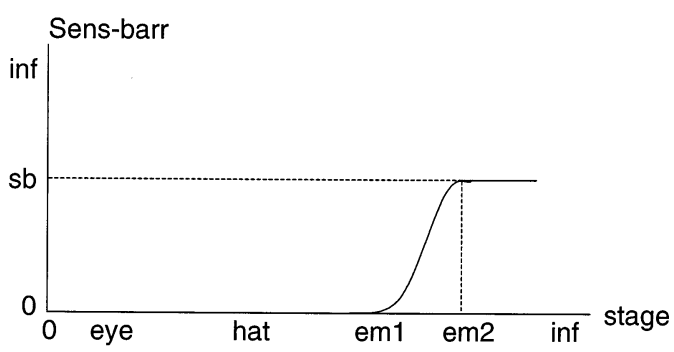

Fig. 7. Graphical representation of the relation holding between Sens-barr (sensitivity of fish to fine deposition) and stage modeled as the $S+$ constraint: $((S+$ stage Sens-barr (em1 0) (em2 sb))).

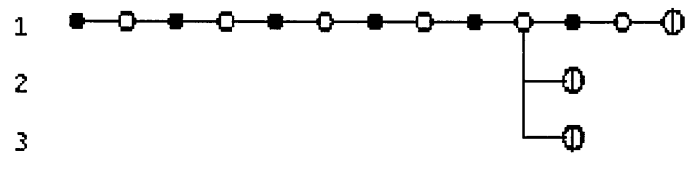

Fig. 8. The behavior tree generated by the slow-QDE simulation, exihibiting three behaviors from a single initial state.

((mult $\left.\left.F_{i} S_{0} \mathrm{dg}\right)(\mathrm{fa} s \mathrm{da})(\mathrm{fb} 1 \mathrm{db})\right)$.

As the intergravel flow velocity $u$ is a monotonic increasing function of both dome and $F_{i}$, these functions are summarized by a single multivariate monotonic constraint $\mathrm{M}++$ (equivalent to two $\mathrm{M}+$ constraints holding simultaneously). It is given in Fig. 17.

Finally, we state that free-O2, the amount of dissolved oxygen available to fish, is a monotonic increasing function of $u$ (Fig. 18).

\subsection{Fast-QDE simulation}

Let us specify an initial state where rain and fines are set to 0 , dome and free-O2 to their upper landmark value $d$ and ob, $Q$ to $\mathrm{qa}$, and $\operatorname{dd} Q$ strictly positive:

$(\mathrm{dg}(\mathrm{db}$ std $))$

$\left(S_{0}(1 \mathrm{std})\right)$

(fines (0 nil))

(dome (d nil))

( $Q$ (qa nil))

$(\mathrm{dd} Q((0$ inf $)$ nil $))$

(rain $(0$ inc) $)$

(free-O2(ob nil)).

We get five distinct behaviors with two branching states (Fig. 19). Let us consider behavior 1 (Fig. 20). Rain remains increasing from zero as the simulation proceeds. According to its time derivative $\mathrm{d} Q$, moving from negative to positive qmags, $Q$ decreases from $T_{0}$ to $T_{1}$, passes by a minimum value $q-0<\mathrm{qa}$, then increases continuously after $T_{1}$ until the simulation ends. Since no transition is specified here, the simulation halts when a state involving variables moving outside 


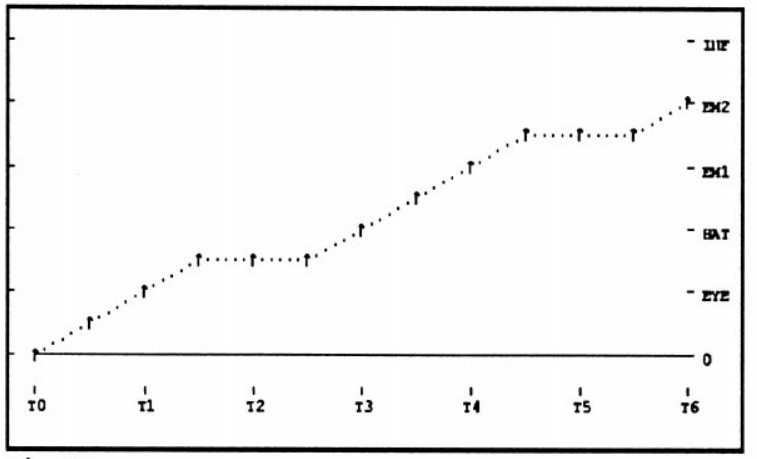

stage

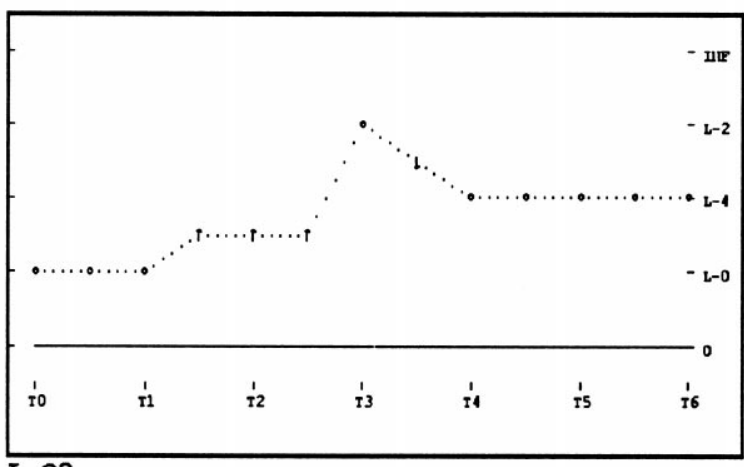

$\mathrm{L}-\mathrm{O2}$
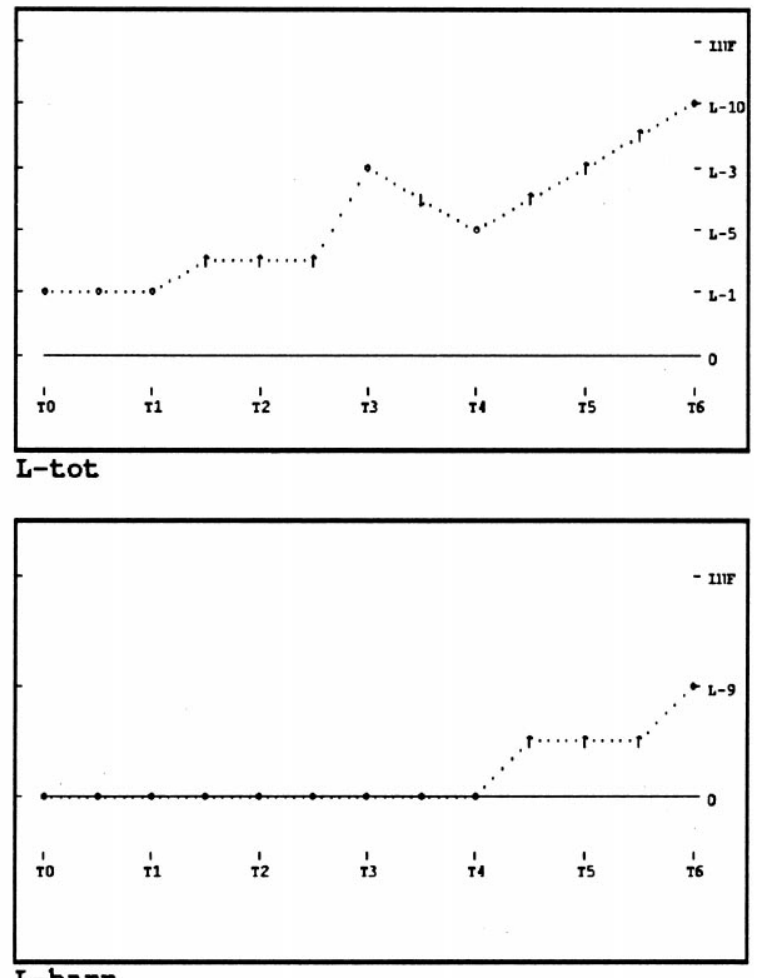

L-barr

Fig. 9. Qualitative plots of behavior 1 generated by the slow-QDE simulation for four variables: development stage of fish (stage), partial $(L-\mathrm{O} 2$ and $L$-barr) and total $(L$-tot) mortality rates.

their range is reached (see for example, $u$ and free-O2, with qualitative values $\langle 0$, dec $\rangle$ at $T_{5}$ ). This delayed evolution of $Q$ with respect to rain is in keeping with modeling the catchment as a linear reservoir (Eq. (5)). Consequently, TSS starts increasing after $Q$ returned to its preceding value qa, i.e. after $T_{2}$. Following dfine, monotonically linked to TSS, fines increases also after $T_{2}$, Fi (according to $\mathrm{d} g$ and $S_{0}$ opposite evolutions) and dome decrease, and so, $u$ and free-O2 tend towards zero.

Comparing all the 5 behaviors, no qualitative difference can be found among several variables such as rain, fines, $\mathrm{Fi}, u$ and free-O2. Therefore, the simulation outputs are not contradictory: focusing on these variables would give a single behavior. Only subtle, interesting, differences appear. For example, if we compare, behaviors 1 and 4 with respect to variables $Q$, TSS and dome
(Fig. 21), one can notice after $T_{2}$ : whereas $Q$, TSS and dome stay within specified bounds (qa, qb), $(\mathrm{sa}, \mathrm{sb})$, and $(0, \mathrm{~d})$ in behavior 1 , in behavior 4 , they cross or reach a bound at $T_{3}(\mathrm{qb}, \mathrm{sb}, 0$, respectively). This is the same phenomenon of occurrence branching like in the slow-QDE behaviors comparison (Figs. 9 and 10). It suggests that the same final result may be obtained by slightly different ways: before some critical values are reached in the case of behavior 1 compared to behavior 4 (e.g. dome need not be 0 for free-O2 to drop to its minimal 0 value).

\section{Coupling slow and fast QDEs}

\subsection{Modifying $Q D E$ structures}

Coupling the slow and fast QDEs consists in bridging them via some shared variables, by 


\section{Behavior 2}
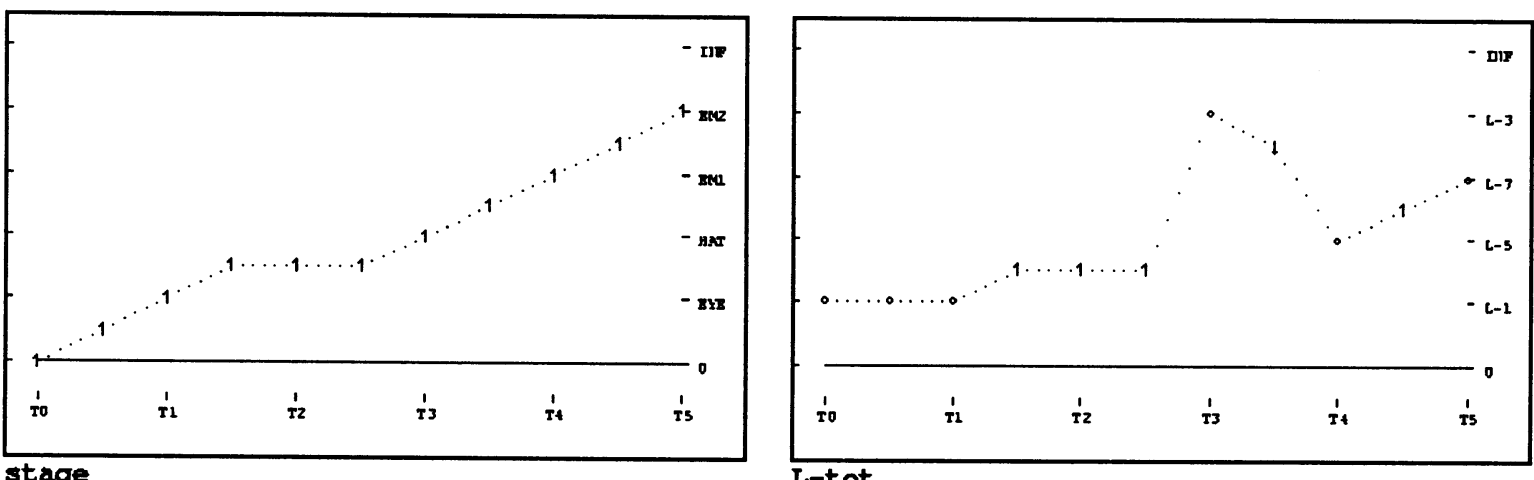

Behavior 3
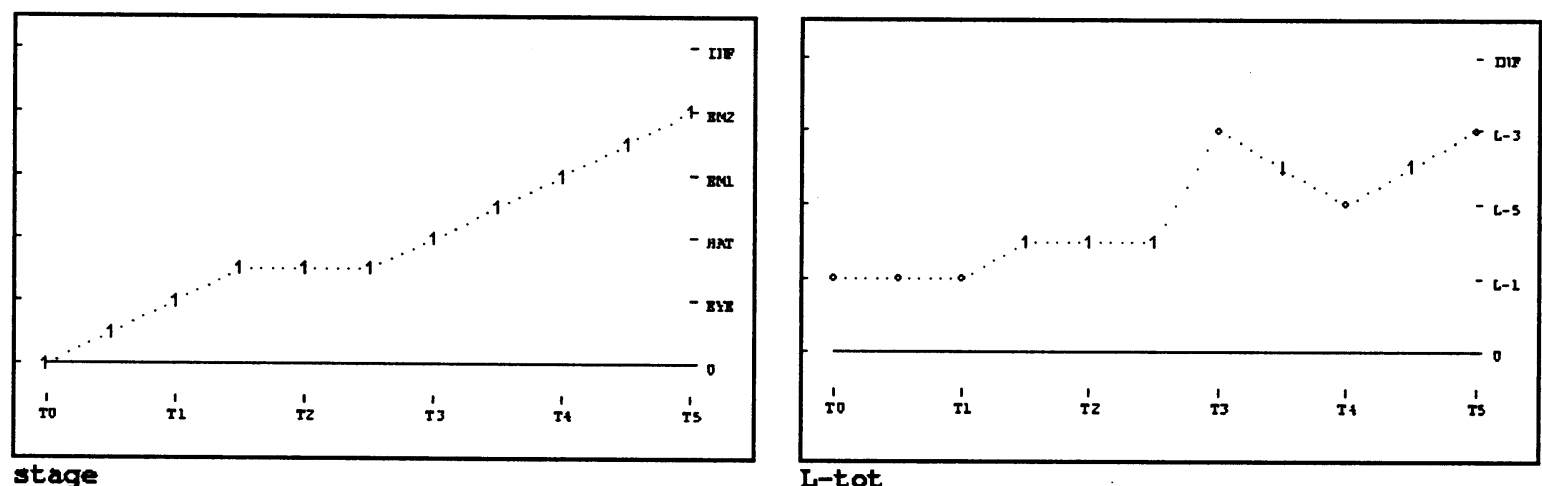

Fig. 10. Comparison of behaviors 2 and 3, generated by the slow-QDE simulation, based on variables stage (development stage of fish) and $L$-tot (total mortality rate).

means of transition states, in order to make alternate simulations of both. It is performed by qualitative transitions that were already used in the clock-QDE to force clock ticks (transitions from the clock-QDE to itself; see Appendix A) and, in the slow-QDE, to halt simulation after emergence has been completed $^{3}$ (see Appendix B).

Here, we want to simulate the slow process evolution and, at some states (e.g. reaching a specific stage like hatching), simulate the occurrence of a rainfall event (by the fast-QDE), that will impact fines and free-O2 magnitudes which,

\footnotetext{
${ }^{3}$ This transition condition is kept in the slow-QDE, here.
}

in turn, will affect the mortality rates of fish ( $L-\mathrm{O} 2$ and $L$-barr) in the slow-QDE. Therefore, we use three shared variables: stage (from the slow-QDE), fines and free-O2 (from the fastQDE). Modifying existing codes for these QDEs is performed through the following steps:

1. Complete each QDE with the quantity spaces of the variables shared with the other:

$\circ$ in the slow-QDE we must add fines and free-O2 qspaces,

- In the fast QDE, the stage qspace.

2. Add constraints for the new shared variables: $O$ in the slow-QDE, we need linking fines to $P$-barr and free-O2 to $P$-O2 by two 
monotonic relations using $\mathrm{M}+$ and $\mathrm{M}-$ constraints, respectively (Fig. 22); we need also to

Table 3

Fast-QDE: qualitative variables and quantity spaces

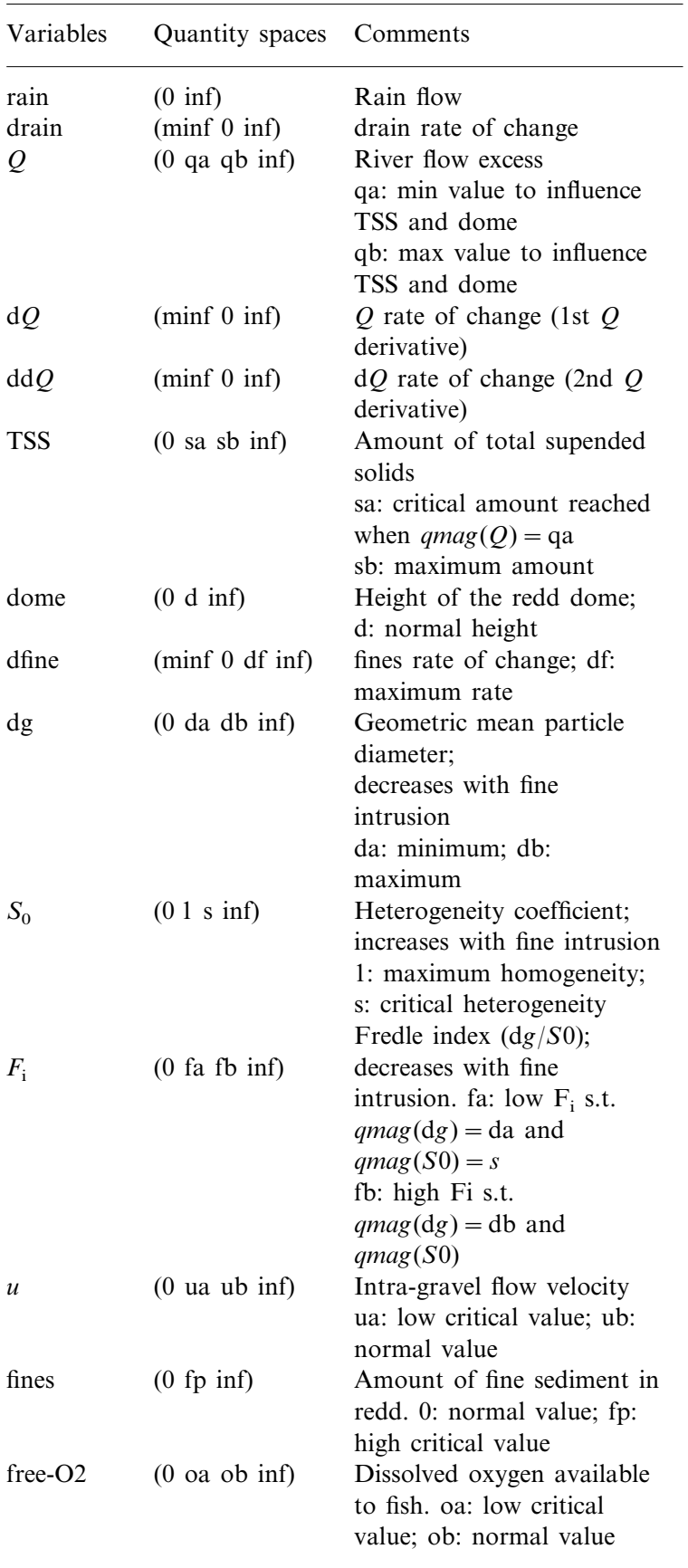

specify fines and free-O2 as constants (external variables stay unchanged during the other QDE simulation);

0 in the fast-QDE, we specify stage as a constant (same reason as above).

3. Specify transition condition $\rightarrow$ transition function mappings:

$\circ$ in the slow-QDE we set the value of stage that must trigger the transition to the fast-QDE, e.g.:

$(($ stage (hat inc)) $\rightarrow$ slow-to-fast)

meaning that after hatching the rainfall event simulation by the fast-QDE must start, ${ }^{4}$

in the fast-QDE we can, e.g. set a condition on the river flow:

$((\mathrm{Q}(\mathrm{qb}$ nil $) \rightarrow$ fast-to-slow $)$

meaning that, whatever its qdir, when $Q$ reaches $\mathrm{qb}$, the fast-QDE simulation must transition to the slow-QDE.

4. Define transition functions (Fig. 23):

when transition is triggered from the slowQDE current state, the slow-to-fast transition function creates a transition state to initialize the fast-QDE simulation, starting with inherited qmags and qdirs for shared variables and values asserted for other variables (*init-fast*, in Fig. 23, is same as the fast-QDE init values given by expression 14);

- similarly, we write the fast-to-slow transition function, where only qmags of free-O2 and fines are inherited, and the next value of stage is asserted.

\subsection{Coupled QDEs simulation}

Hence, alternate simulations of both slow and fast QDEs are performed, according to time-scale abstraction principles (Kuipers, 1994):

- the slower process views the faster one as instantaneous: the continuation of the slow-QDE

\footnotetext{
${ }^{4}$ Substituting hat with other landmarks of stage qspace would trigger the transition at other stages; if wished, several successive transitions can also be set.
} 


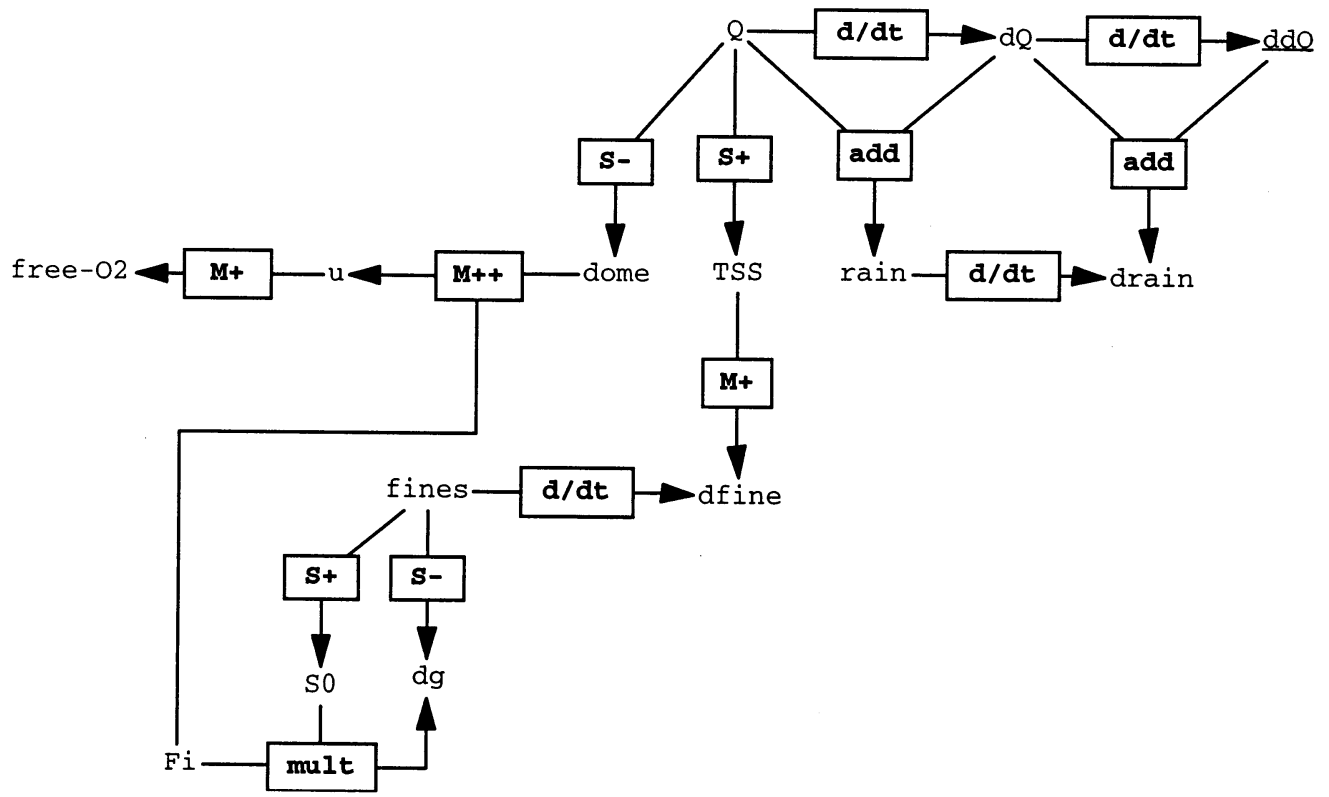

Fig. 11. Graphical representation of the fast-QDE (same legend as in Fig. 1).

simulation is performed after updating its latest state reached before the transition;

- the faster process views the slower one as constant: all the variables of the slow-QDE are frozen (their qdirs are set to std) while the fast-QDE is simulated.

The impact of a rainfall event is presented, here, as an example. It starts by the clock-QDE simulation, providing critical dates for stage changes in order to simulate the slow-QDE from an initial state (same as in expression (4)), and using the transition functions (Fig. 23) to initiate the fast-QDE simulation (see Fig. 24 presenting a synthetic view of how simulations are performed).

We get a tree with 31 behaviors (Fig. 25) issued from the combination of branching in the slow and fast-QDEs (Figs. 8 and 19). As we already dealt with multiple behaviors comparison before (Sections 3 and 4), we will not emphasize this now. We focus here on describing how are performed alternate simulations of both QDEs. To illustrate this, we consider behavior 2. Fig. 26 summarizes both categories of co-evolutions of the variables during the rainfall episode $\left(T_{3}-T_{8}\right)$ : rain increases which makes $Q$ increase (after a delay due to linear reservoir storage), TSS and fines increase while dome and free-O2 diminish. The interpretation of mortality is a bit more complex since it is under both influences of sensitivities to lethal factors and those factors pressures. However, the fact that $L-\mathrm{O} 2$ stabilizes at a higher level at $T_{9}$ compared to its value preceding the rainfall episode at $T_{3}$ and that $L$-tot grows after $T_{9}$, denote the negative influence of the rainfall episode, because of fines accumulation within the redd, on both mortality factors.

Typically, such cascading simulations are performed as follows (otherwise specified, conments refer to Fig. 26):

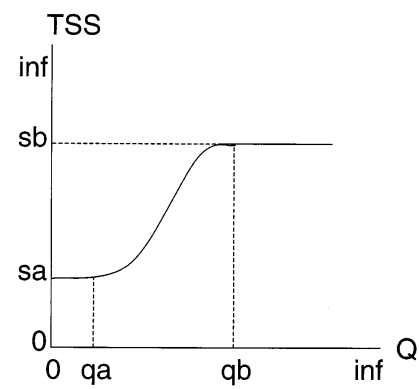

Fig. 12. Graphical representation of the relation holding between TSS (total suspended solids) and $Q$ (excess in river flow) modeled as the $S+$ constraint: $((S+Q$ TSS (qa sa)(qb sb))). 


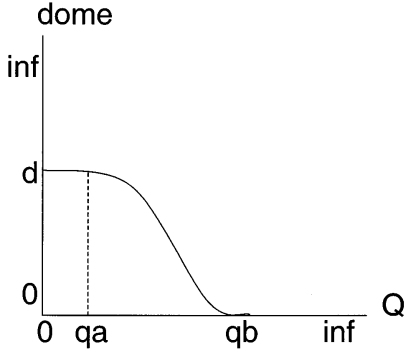

Fig. 13. Graphical representation of the relation holding between dome (i.e. the redd height) and $Q$ (excess in river flow) modeled as the $S-$ constraint: $(S-Q$ dome (qa d)(qb 0$)$ )).

1. Specification by the user of:

- a temperature regime, as a sequence of average water temperatures (Section 2),

$\circ$ events, as transition conditions $\rightarrow$ transition functions mappings (Section 5.1),

- initial states of both QDEs (here unchanged compared to expressions (4) and (14)).

2. Clock simulation:

$\circ$ as previously described Section 2, thus generating the critical dates for stage changes.

3. Slow-QDE simulation:

$\circ$ completion of the initial state and generation of qualitative behaviors until a transition condition is reached;

- from $T_{0}$ to $T_{3}$ : mortality rates $(L-\mathrm{O} 2, L$ barr, $L$-tot) evolve according to the evolution of the sensitivities to mortality factors that are influenced by the growth of the developmental stage of fish.

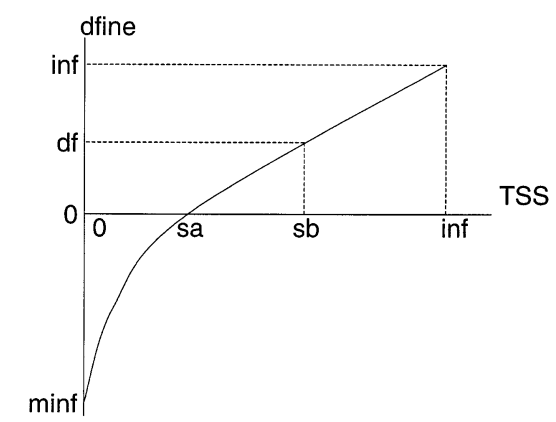

Fig. 14. Graphical representation of the relation holding between dfine (i.e. fine intrusion rate of change) and TSS (amount of total suspended solids) modeled as the $\mathrm{M}+$ constraint: ((M+ TSS dfine) (0 minf) (sa 0) (sb df) (inf inf)).

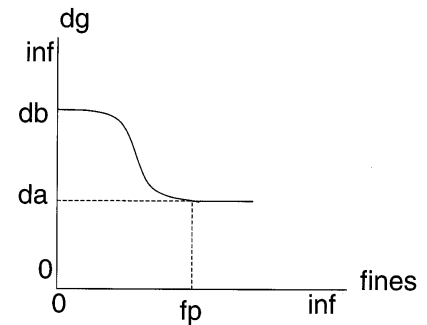

Fig. 15. Graphical representation of the relation holding between dg (geometric mean diameter of gravels) and fines (amount of fine particles in the redd) modeled as the $S$ constraint: $((S-$ fines dg $(0 \mathrm{db})(\mathrm{fp} d a)))$.

4. Transition slow-QDE $\rightarrow$ fast-QDE:

$\circ$ at $T_{3}$, corresponding to the date $n-6$, i.e. 70th day since spawning, hatching is reached (condition for triggering the slowto-fast transition), and so $L$-O2 and $L$-tot are maximal;

- qualitative values of shared variables in the transition state of the slow-QDE (stage, fines, free- $\mathrm{O} 2^{5}$ ) are passed to the initial state of the fast-QDE;

- variables of the slow-QDE are frozen (see $L$-O2, $L$-barr, $L$-tot for which plots are left blank in the $T_{3}-T_{8}$ interval).

5. Fast-QDE simulation:

o initial state completion and generation of qualitative behaviors until a transition condition is reached;

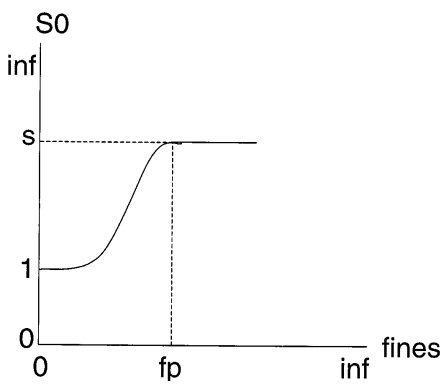

Fig. 16. Graphical representation of the relation holding between $S_{0}$ (heterogeneity coefficient of the redd structure) and fines (amount of fine particles in the redd) modeled as the $S+$ constraint: $\left(\left(S+\right.\right.$ fines $\left.\left.S_{0}\right)(01)(f p ~ s)\right)$.

\footnotetext{
${ }^{5}$ The latter two variables only are represented on Fig. 26.
} 

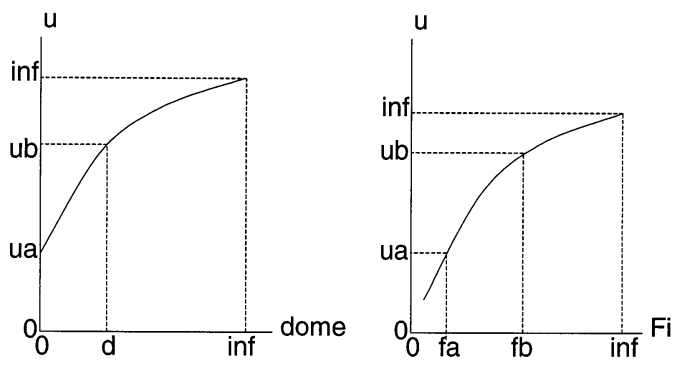

Fig. 17. Graphical representation of the relation holding between $u$ (waterfiow within the redd) and both dome (redd height) and $F_{\mathrm{i}}$ (Fredle index) modeled as the $\mathrm{M}++$ constraint: $\left(\left((\mathrm{M}++)\right.\right.$ dome $\left.F_{\mathrm{i}} u\right)(0 \mathrm{fa} \mathrm{ua})(\mathrm{d}$ fb ub) (inf inf inf $\left.)\right)$.

from $T_{3}$ to $T_{8}$ : a sudden rainfall episode is triggered at $T_{3}$, provoking an increase in the river flow $Q$ after $T_{4}$ which makes TSS increase after $T_{5}$, along with a progressive accumulation of fines in the redd and dome erosion, reducing accordingly the water flow through the gravel layers and, consequently, the oxygen available to alevins (free-O2).

6. Transition fast-QDE $\rightarrow$ slow-QDE:

$\circ$ at $T_{8}: Q$ reaches qb (transition condition);

- qualitative values of shared variables in the transition state of the fast-QDE are passed to the initial state of the slow-QDE;

- fast-QDE variables are frozen (see rain, $Q$, $\mathrm{TSS}$, fines, dome, and free-O2 for which plots are left blank after $T_{8}$ as well as before $\left.T_{3}\right)$.

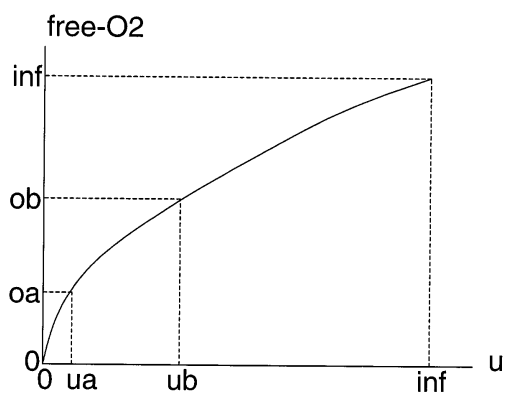

Fig. 18. Graphical representation of the relation holding between free-O2 (dissolved oxygen available to fish) and $u$ (waterflow in the redd) modeled as the $\mathrm{M}+$ constraint: $((\mathrm{M}+u$ free-O2) (0 0) (ua oa) (ub ob) (inf inf)).

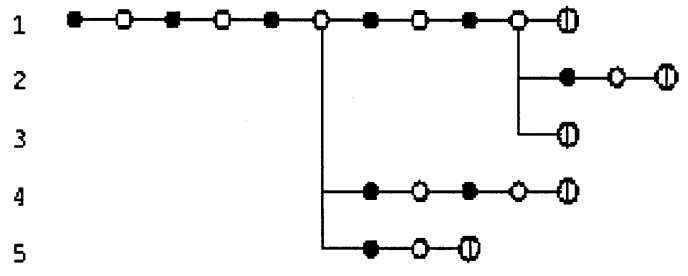

Fig. 19. The behavior tree generated by the fast-QDE simulation exhibiting five behaviors from a single initial state.

7. Continuation of the slow-QDE simulation:

0 the new initial state of the slow-QDE is made from the update of its latter state frozen at the previous transition $T_{3}$ (date and stage restart right after landmarks $n-6$ and hat, respectively);

- back to the slow-QDE simulation from $T_{8}$ to $T_{12}$ : stage, $L$-O2, $L$-barr and $L$-tot, pursue their evolution from their values left at $T_{3}$.

8. Simulation halts when limits of the validity domain of the model are reached, e.g. (em2 inc) for stage.

\section{Discussion and perspectives}

\subsection{Validation/verification of the redd qualitative model}

Due to the strong variability and uncertainty characterizing field or laboratory data highlighted by Lisle and Lewis (1992), it seems hard to envisage a 'classical' validation of such a model as a quantitative comparison between the model outputs and measured numerical data. This is indeed a big factor limiting the feasibility and interest of quantitative approaches in a domain such as salmonid ecology. For example, van Winkle et al. (1998) show that available field data (trout abundance estimates) used to calibrate their quantitative model exhibited variation coefficients ranging from 75 to $255 \%$. Therefore, even fitting their simulation outputs with the data used to calibrate the model is not that easy, so that the validation criterion used by these authors is as vague as 'model predictions and field observations should be in reasonable agreement' (van Winkle et al., 

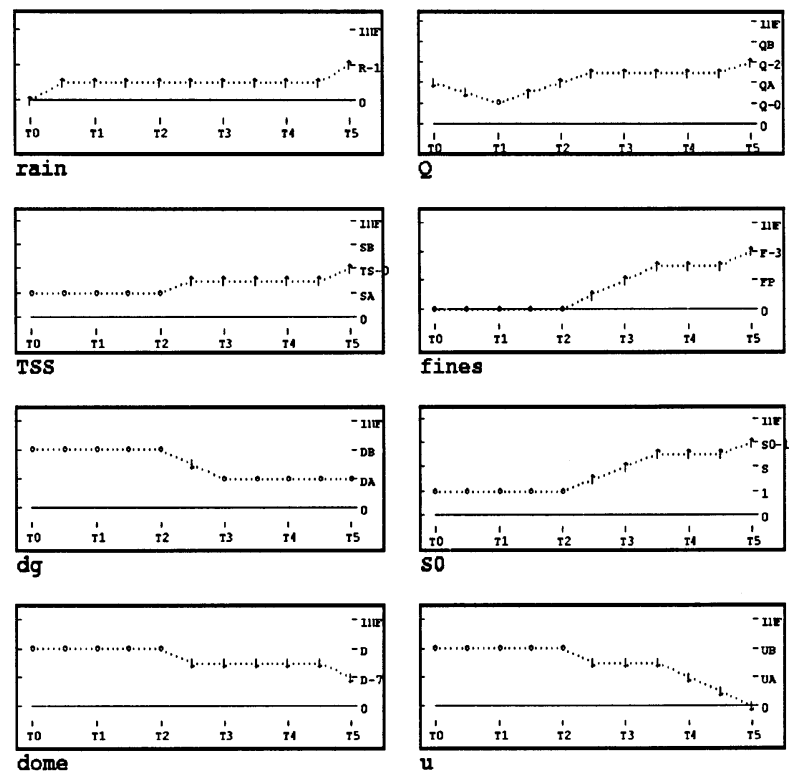
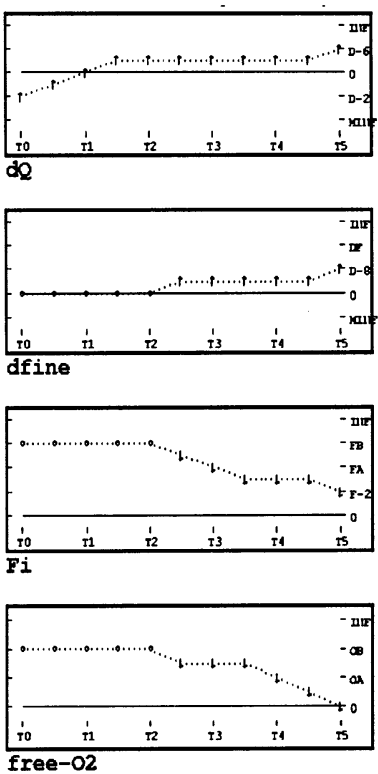

Fig. 20. Qualitative plots of behavior 1 generated by the fast-QDE simulation.

1998). Jessup (1998) also, 'validates' his quantitative model by considering only the coarse shape similarity of curves accounting for historical data and simulation outputs, but disregarding the large numerical discrepancies between both categories. These examples are, indeed, kinds of qualitative validation.

These considerations were for us a strong encouragement in attempting to use qualitative modeling and simulation to represent the functioning of redds and the influences of physical variables on embryos, alevins and fry mortality. As stated by Chapman (1988), who did a large review of the scientific literature (involving more than seventy articles of various authors) on the effects of fines in salmonid redds: 'One cannot, with existing information on survival of embryos and alevins in the redds of large salmonids, predict survival quantitatively and with known accuracy on the basis of physical factors measured in field or laboratory studies'. However, in the same paper, the author remarks that there exists, at least, a consensus in the literature to consider that survival to alevin emergence:

1. relates positively to geometric mean particle diameter, fredle index, permeability and dissolved oxygen in intragravel water;
2. relates negatively to percentages of fines in redd.

Since our principal goal was to represent qualitatively existing knowledge, we find correct to compare the model outputs with the general expertise expressed either by Chapman's (1988) consensual statements or by finer relations holding between physical and biological variables as those described by Lisle and Lewis (1992). This complies with Rykiel's (1996) conception of validation, according to which validating a model means checking its use against a reference system, be it an actual (physical) system, another model or, even, human expertise, according to specified criteria. $^{6}$

With this respect, our qualitative models outputs comply with Chapman's conclusions; e.g. the behaviors obtained from the fast-QDE simulation (Fig. 20) show that $\mathrm{d} g, \mathrm{Fi}$, and free-O2 (inversely related to the mortality rate $L-\mathrm{O} 2$ in the slowQDE) decrease when fines increase. They are also qualitatively consistent with all the empirical rela-

6 "Validation means that a model is acceptable for its intended use because it meets specified performance requirements" (Rykiel, 1996). 


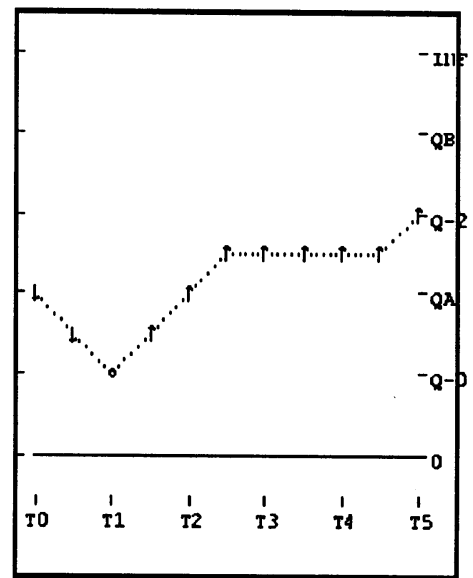

Beh 1 Var $Q$

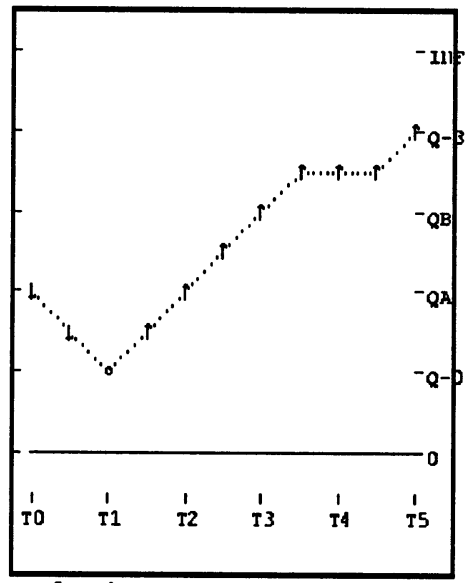

Beh 4 Var $Q$

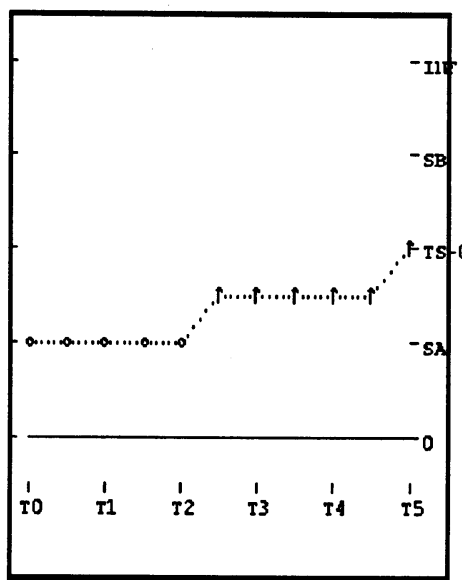

Beh 1 Var TSS

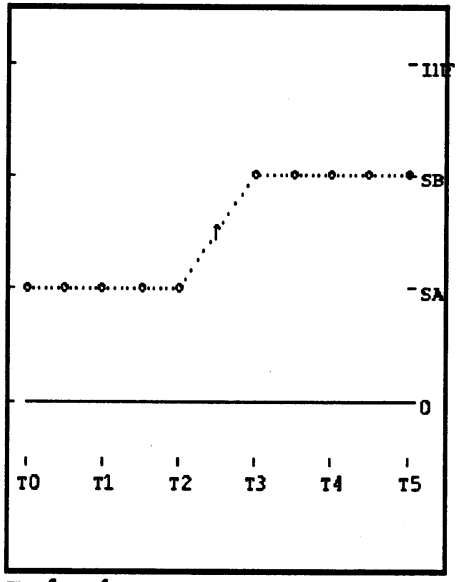

Beh 4 Var TSS

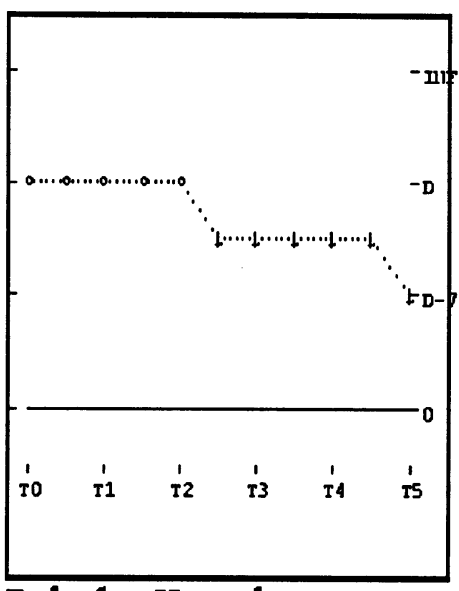

Beh 1 Var dome

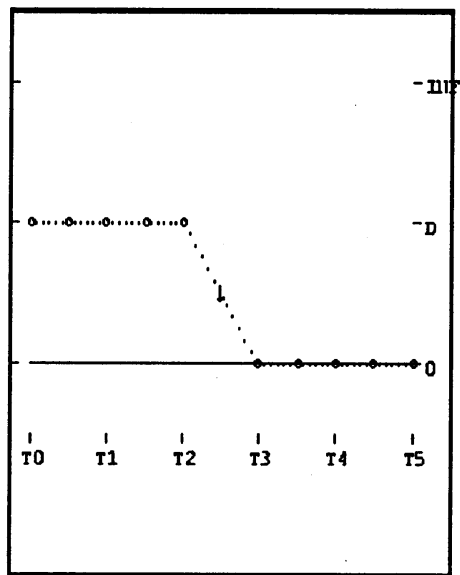

Beh 4 Var dome

Fig. 21. Comparison of behaviors 1 and 4 generated by the fast-QDE simulation, based on variables $Q$ (river flow excess), TSS (total suspended solids carried out by the river), and dome (height of the redd dome).

tions (quantitative) given by Lisle and Lewis (1992):

- fine-sediment infiltration grows with the finesediment transport rate which increases with the water discharge: e.g. this is illustrated on Fig. 20 where fines grows with TSS (from $T_{2}$ to $T_{5}$ ) which grows in turn with $Q$ for $\operatorname{qmag}(Q) \geq \mathrm{qa}$;

- diameter of particles in the redd is reduced by fine-sediment infiltration: e.g. this is verified by the evolution of $\mathrm{dg}$ which decreases when fines increases (Fig. 20);

- survival decreases when fine-sediment infiltration increases and intergravel flow velocity de- creases (due to the decrease in the redd permeability): e.g. this is illustrated on Fig. 20 by the evolutions of fines, free- $\mathrm{O} 2$ and $u$.

The interpretation given Section 5.2 of the coupled-QDEs simulation is also consistent with the explanations above.

Therefore, our model can be 'validated' with respect to the criterion of enabling us to represent consistently expert knowledge. ${ }^{7}$ But it is clear that

\footnotetext{
${ }^{7}$ This is, of course, a weak criterion as is weak the available knowledge on redds (and, more generally, on complex natural systems) compared to most of physical, easy-to-measure, manmade systems.
} 

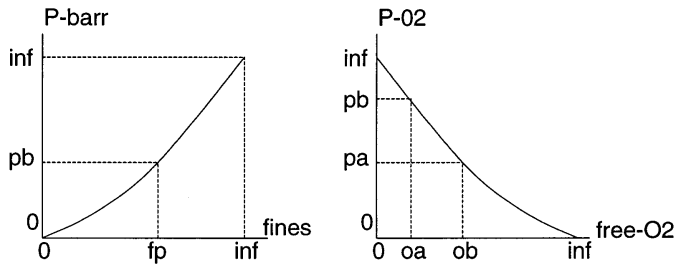

Fig. 22. Linking both QDEs by means of shared variables $P$-barr and $P$-O2 (slow-QDE), fines and free-O2 (fast-QDE), using $\mathrm{M}+$ (left) and $\mathrm{M}-$ (right) constraints: $((\mathrm{M}+$ fines $P$-barr) $(00)(\mathrm{fp} \mathrm{pb})($ inf inf) $)$ and $((\mathrm{M}-$ free-O2 $P$-O2) $(0$ inf $)$ (oa pb) (ob pa) (inf 0$)$ ).

it is far from being validated as a management tool. Our work also demonstrates globally the adequacy of Qsim to account both for available analytical knowledge (like performing abstraction of ODEs into QDEs Section 4.1) and for informal expertise. Qsim allowed us to simulate relevant behaviors of such systems, by handling a relatively large number of variables by means of model decomposition. This aspect is close to the verification concept, i.e. to demonstrate that the modeling formalism is correct (Rykiel, 1996).

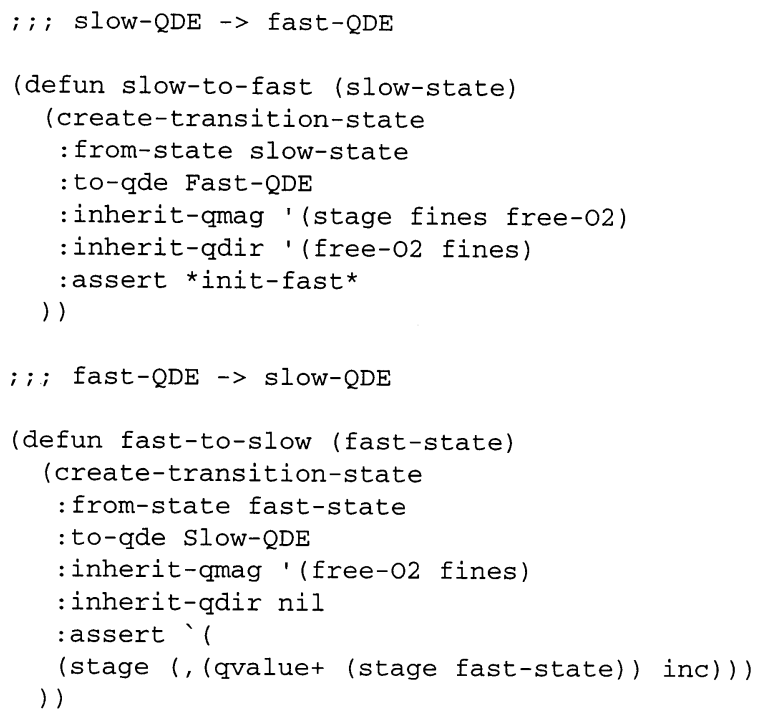

Fig. 23. Coupling slow and fast QDEs: transition functions from the slow-QDE to the fast-QDE (top) and conversely (bottom)

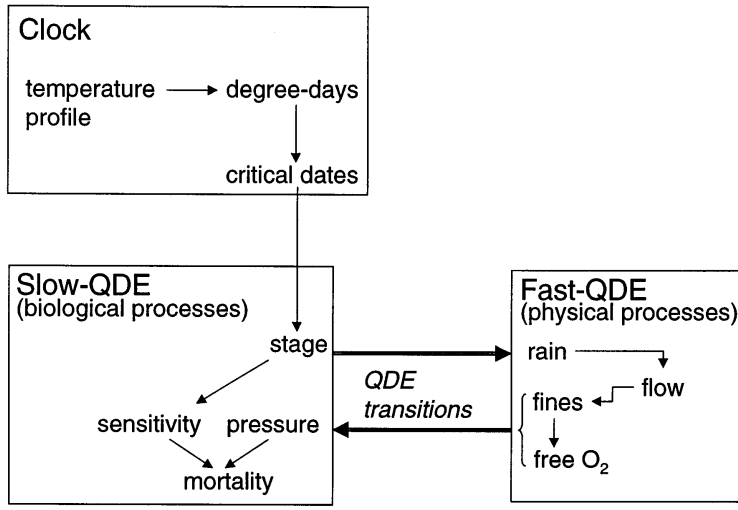

Fig. 24. General description of alternate simulations involving the clock-QDE and the coupled slow and fast QDEs via qualitative transitions, based on shared variables (stage, fines and free-O2).

\subsection{Perspectives}

Our salmon redd model could be enriched, for example by introducing other mortality factors like shocks, water temperature, influence of the female size, superimposition of redds, dewatering, and ... unspecified causes (van Winkle et al., 1998). However, in order to come closer to a decision tool, which needs to understand and simulate more completely how human activities impact on redds, it can be sought to model the relations between:

- the river catchment characteristics, both in terms of geographical features and of human activities impacting on erosion processes or TSS generation;

- the river hydrology and its impact on redds according to their locations.

Therefore, beyond the fry population and the redds, two other blocks of processes (catchment and river levels; see Fig. 27) need be distinguished as they relate to distinct spatial scales and pose different modeling problems.

At the catchment level, semi-quantitative (e.g. intervals) to ordinal, or even nominal, data are used to describe elementary features. For example, slopes, lithology, and land use are the indicators that were taken in order to map the erosivity of sub-catchments of the Nivelle river. Erosivity is expressed as five qualitative classes ranging from 
very-weak up to very-strong (de Maisonneuve et al., 1997). Similarly, slope classes are usually expressed as numerical percentage intervals and soil type are nominal data: e.g. clay, silt, sand (Botterweg et al., 1998). In some cases, empirical or black-box models may exist, like statistical regressions between rainfall and river flow, or hydrologic mechanistic models describing surface runoff and erosion in the catchment or TSS and sediment transportation by the river flow. Although, it is difficult to use these models in a fully, precise, numerical way (as explained Section 1), it could be attempted to make use of them more or less qualitatively. For this, it could be worth exploring the approaches followed to introduce numerical information into QDEs to perform semi-quantitative reasoning:

- the static envelope approach, where landmarks and monotonic constraints in QDEs are bounded with upper and lower numerical bounds, which are propagated through the constraints, based on interval arithmetic (Kuipers and Berleant, 1988; Berleant and Kuipers, 1990);

- the dynamic envelope approach, where QDEs are bounded by envelopes determined by ex-

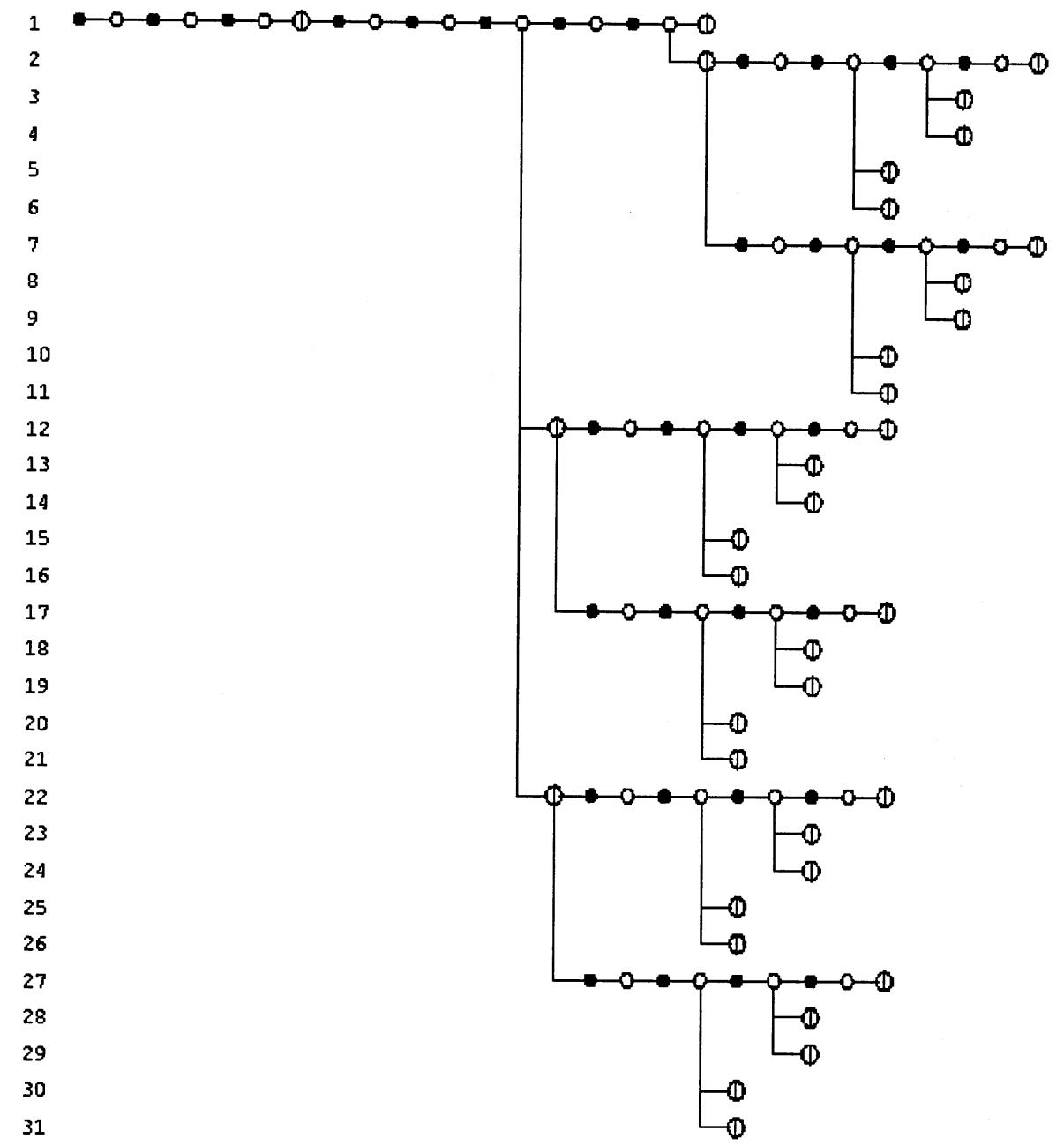

Fig. 25. The behavior tree generated by coupling simulations of slow and fast QDEs, exhibiting 31 behaviors from a single initial state. 


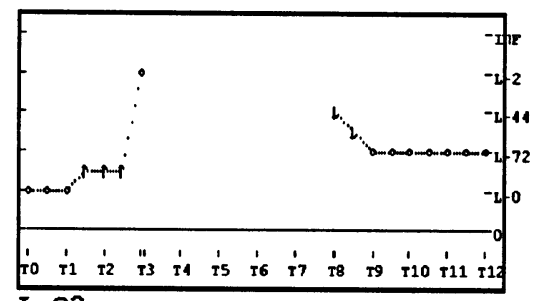

$\mathbf{L}-\mathbf{0 2}$

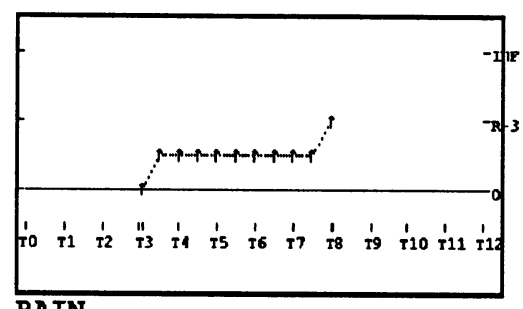

RAIN

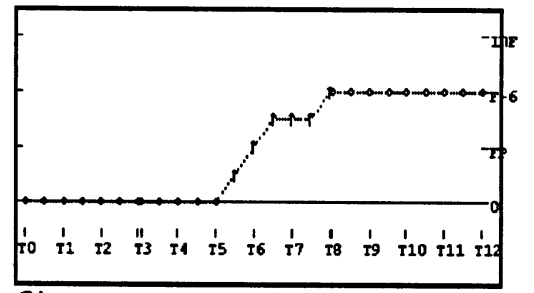

fines

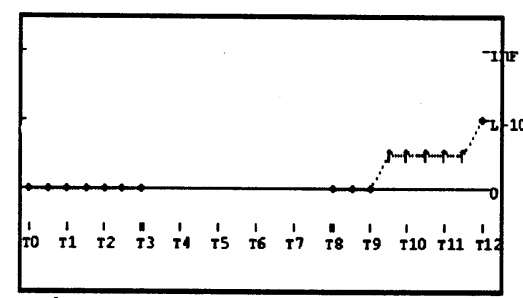

L-barr
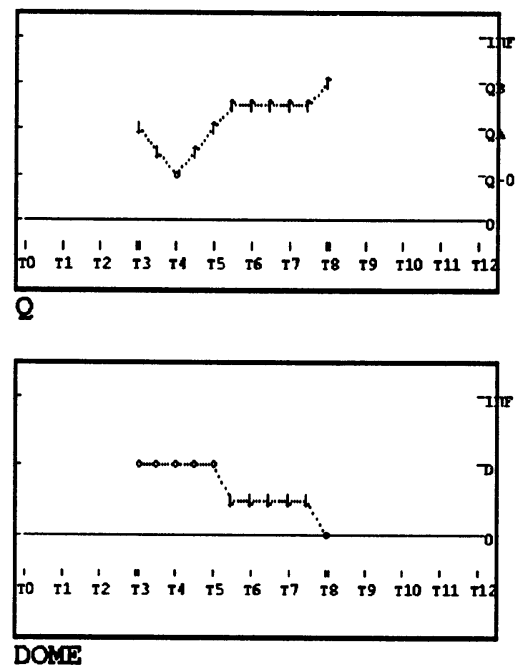

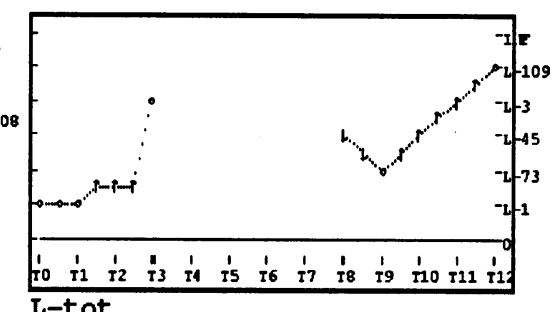

L-tot

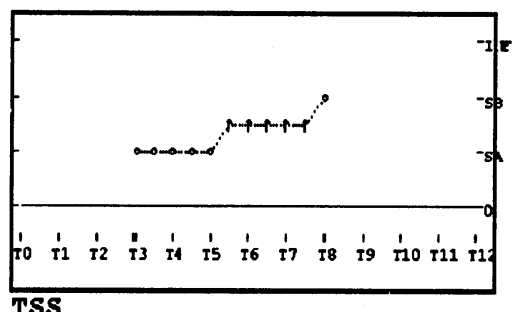

TSS

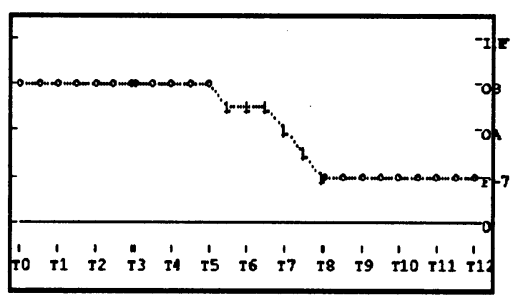

free-02

Fig. 26. An example of simulated behavior from coupling slow and fast QDEs. The slow-QDE is simulated from $T_{0}$ to $T_{3}$, and $T_{8}$ to $T_{12}$; the fast-QDE is simulated from $T_{3}$ to $T_{8}$. Absence of plotting in variable plots for some periods is explained by alternate simulation of QDEs; shared variables only (here, fines and free-O2) have continuous plots.

tremal ODEs, numerically integrated (Kay, 1996).

Both approaches restrict the set of generated behaviors to those consistent with both qualitative and numerical constraints, improving so the simulation accuracy.

Connecting our redd qualitative model outputs to a salmon population dynamics quantitative model has been envisaged (Badia et al., 1996). Again, this perspective raises several problems of interfacing symbolic/numeric representations. Very often, mixed types of elements should be combined. For example, survival rates of, say, $\approx 100 \%$ at spawning, $\approx 90 \%$ at the eyed-embryo stage, $\approx 60 \%$ at hatching, $\approx 40 \%$ at the end of fry emergence, will be all termed excellent by freshwater ecologists. Similarly, for each stage, experts can define approximate percentages corre- sponding to labels such as good, average, mediocre, bad. Therefore, beyond the way it is dealt with that kind of problems in the static envelope approach (Kuipers and Berleant, 1988), that is mapping (by hand) symbolic landmarks

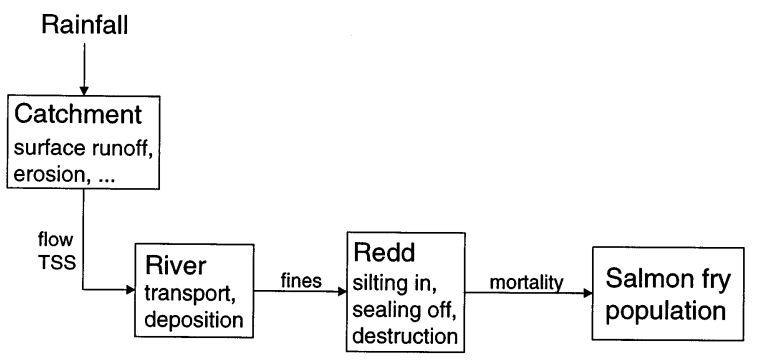

Fig. 27. Blocks of processes to be considered, in order to assess the impact of the environment on a fry population of salmon at several levels of organization. 
with numerical intervals, the question is posed here how to automatically perform more complex mappings such as (symbol, number) $\mapsto$ symbol, and to combine many different types of such mappings within the same modeling framework. In modeling the impact of land use (mainly construction) on brown trout population dynamics, an interesting quantitative approach has already been undertaken in that sense. It uses a continuous $[0,1]$ scale (as Habitat Suitability Indices) to translate numerical data (watershed characteristics, land use, etc.) into signifying weighted influences on biological variables (Jessup, 1998). Along with other aspects of quantitative/qualitative integration, this can be a valuable research perspective.

\section{Acknowledgements}

This work was performed at Unité de Biométrie et Intelligence artificielle (Inra, Toulouse, France) with freshwater ecologists of Station d'Hydrobiologie (Inra, Saint-Pée-sur-Nivelle, France). We are grateful to R. Faivre and J. Badia (from Inra, Toulouse) for useful comments about the use of qualitative simulation and participation in tracing future perspectives. We are also indebted towards P. Davaine, E. Beall and O. Clément (from Inra, St-Pée-sur-Nivelle) for contributing in expertise transfer about salmon ecology.

\section{Appendix A. The clock-QDE}

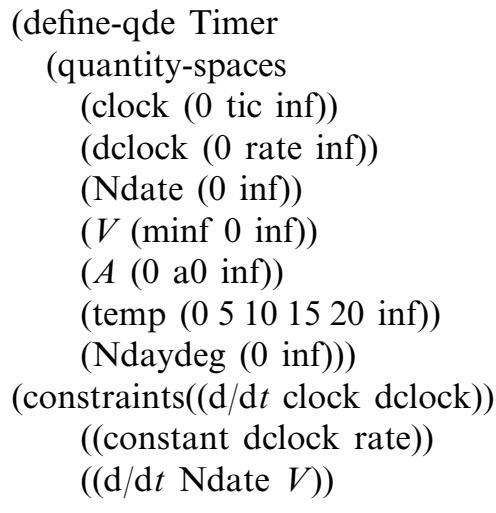

\author{
$((\mathrm{d} / \mathrm{d} t V A))$ \\ ((constant $A))$ \\ ((constant temp)) \\ ((constant Ndaydeg))) \\ $($ transitions $(($ clock $($ tic inc $)) \rightarrow$ tac) $))$
}

Appendix B. The slow-QDE

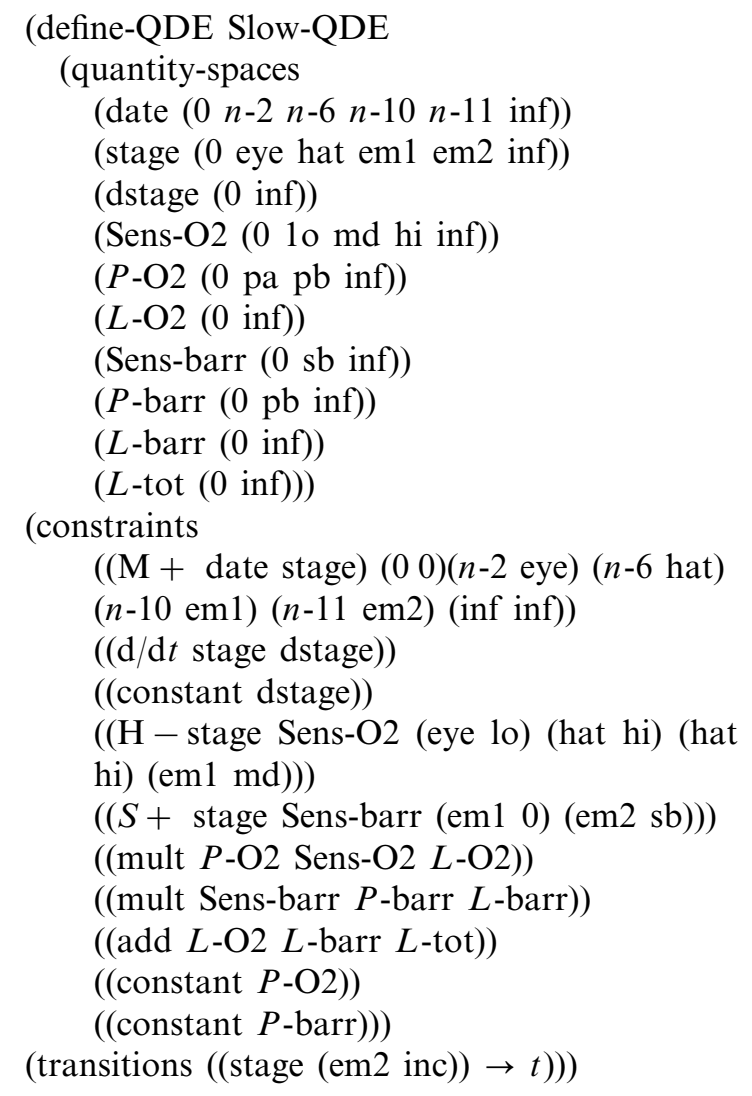

\section{Appendix C. The fast-QDE}

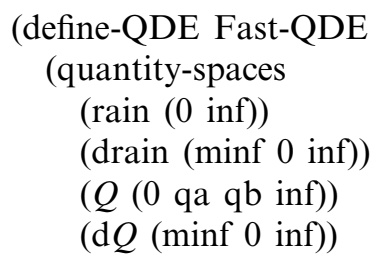


(dd $Q$ (minf 0 inf) $)$

(TSS (0 sa sb inf))

(dome $(0 \mathrm{~d}$ inf))

(dfine (minf 0 df inf))

(dg (0 da db inf))

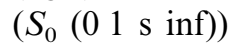

$\left(F_{\mathrm{i}}(0 \mathrm{fa} \mathrm{fb}\right.$ inf $\left.)\right)$

( $u$ (0 ua ub inf $))$

(fines $(0 \mathrm{fp}$ inf))

(free-O2 (0 oa ob inf)))

(constraints

$((\mathrm{d} / \mathrm{d} t Q \mathrm{~d} Q))$

$((\mathrm{d} / \mathrm{d} t Q \operatorname{dd} Q))$

$((\mathrm{d} / \mathrm{d} t$ rain drain $))$

((add $\mathrm{d} Q Q$ rain))

((add $\mathrm{dd} Q \mathrm{~d} Q$ drain))

((constant $\mathrm{dd} Q))$

$((S+Q$ TSS (qa sa) (qb sb)))

$((S-Q$ dome (qa d)(qb 0$)))$

$((\mathrm{M}+\mathrm{TSS}$ dfine $)(0 \mathrm{minf})(\mathrm{sa} 0)(\mathrm{sb} \mathrm{df})(\mathrm{inf}$

inf))

$((\mathrm{d} / \mathrm{d} t$ fines dfine $))$

$((S-$ fines dg $(0 \mathrm{db})(\mathrm{fp} \mathrm{da})))$

$\left(\left(S+\right.\right.$ fines $\left.\left.S_{0}\right)(01)(\mathrm{fp} \mathrm{s})\right)$

((mult $\left.\left.F_{\mathrm{i}} S_{0} \mathrm{~d} g\right)(\mathrm{fa} \mathrm{s} \mathrm{da})(\mathrm{fb} 1 \mathrm{db})\right)$

$\left(\left((\mathrm{M}++)\right.\right.$ dome $\left.F_{\mathrm{i}} u\right)(0$ fa ua)(d fb ub)

(inf inf inf))

$((\mathrm{M}+u$ free-O2)(0 0)(ua oa) (ub ob) (inf inf))))

\section{References}

Badia, J., Faivre, R., Guerrin, F., 1996. Modélisation de grands systémes biologiques. Rapport UBIA 1996/1, Inra, Département de Biométrie et d'Intelligence artificielle, Toulouse, France.

Berleant, D., Kuipers, B., 1990. Qualitative-quantitative simulation with Q3. In: Working papers of the 4th International Workshop on Qualitative Physics (QPW'90), Lugano, Switzerland, pp. 140-152.

Botterweg, P., Leek, R., Romstad, E., Vatn, A., 1998. The Eurosem-Gridsem modeling system for erosion analyses under different natural and economic conditions. Ecological Modelling 108, 115-129.

Chapman, D., 1988. Critical review of variables used to define effects of fines in redds of large salmonids. Transactions of the American Fisheries Society 117 (1), 1-21.

Clancy, D., Kuipers, B., 1997. Model decomposition and simulation: a component based qualitative simulation algorithm. In: Proceedings of 14th National Conference on Artificial Intelligence (AAAI'97), Providence, RI, USA, pp. $118-124$.

Crisp, D., 1993. The environmental requirements of salmon and trout in freshwater. Freshwat. Forum 3, 176-202.

de Maisonneuve, L., Clément, O., Maneux, E., Durnas, J., 1997. Evaluation des risques de colmatage du lit mineur d'une riviére dans une optique de gestion des ressources aquatiques vivantes. In: Les bassins versants expérimentaux de Draix, laboratoire d'étude de l'érosion en montagne. Cemagref, Grenoble, Draix, Le Brusquet, Digne, France, pp. 211-221.

Dumas, J., Darolles, V., 1999. Caractéristiques environnementales et survie embryolarvaire du saumon atlantique, Salmo salar L., dans un cours d'eau du piémont pyrénéen, la Nivelle (France). Cybium 23 (1), 29-44.

Dumas, J., Faivre, R., Charron, M.-H., Badia, J., Davaine, P., Prouzet, P., 1996. Modélisation stochastique du cycle biologique du saumon atlantique (Salmo salar L.): bases biologiques, implémentation informatique et interprétation. In: Ferraris, J., Pelletier, D., Rochet, M. (Eds.), Méthodes d'étude des systémes halieutiques et aquacoles. Vol. 2 of Colloques et séminaires. Orstom éditions, Bondy, France, pp. 211-218.

Dumas, J., Guerrin, F., Darolles, V., Estournés, G., de Maisonneuve, L., 1998. Survie embryolarvaire du saumon atlantique en relation avec les caractéristiques des frayéres et le potentiel d'émission en éléments fins du bassin versant. In: Le poisson dans les zones ateliers, 2ème réunion annuelle du réseau Zones Ateliers. GIP Hydrosystémes, Biarritz, France, pp. 36-43.

Faivre, R., Dumas, J., Charron, M., Badia, J., Prouzet, P., 1997. River basin management using a stochastic model of the salmon life cycle. In: Proceedings of International Congress of Modeling and Simulation (MODSIM'97), Hobart, Tasmania, pp. 1536-1541.

Fouché, P., 1992. Towards a unified framework for qualitative simulation. PhD thesis, Université de Technologie de Compiégne, Compiégne, France.

Guerrin, F., Dumas, J., Davaine, P., Beall, E., Clément, O., 1997. Qualitative modeling of the impact of the environment on early stages of salmon populations. In: Proceedings of 11th International Workshop on Qualitative Reasoning (QR'97), Cortona, Italy, pp. 257-263.

Haan, C., Johnson, H., Bratensiek, D., 1982. Hydrologic modeling of small watersheds. No 5 in ASAE Monograph. ASAE, St Joseph, MI, USA.

Harris, G., 1978. Salmon propagation in England and Wales. Technical report, National Water Council.

Hobbs, D., 1937. Natural production of quinnat salmon, brown and rainbow trout in certain New Zealand waters. New Zealand Mar. Department Fishery Bull. 6, 1-104.

Jessup, B., 1998. A strategy for simulating brown trout population dynamics and habitat quality in an urbanizing watershed. Ecological Modelling 112, 151-167.

Kay, H., 1996. Refining imprecise models and their behaviors. $\mathrm{PhD}$ thesis, The University of Texas at Austin, USA. 
Kuipers, B., 1994. Qualitative reasoning. Modeling and simulation with incomplete knowledge. The MIT Press, Cambridge, MS, USA.

Kuipers, B., Berleant, D., 1988. Using incomplete quantitative knowledge in qualitative reasoning. In: Proceedings of 6 th National Conference on Artificial Intelligence (AAAI-88), Saint-Paul, MN, USA, pp. 324-329.

Lisle, T., Lewis, J., 1992. Effects of sediment transport on survival of salmonid embryos in a natural stream: a simulation approach. Can. J. Fish. Aquat. Sci. 49, 2337-2344.

Lotspeich, F., Everest, F., 1981. A new method for reporting and interpreting textural composition of spawning gravel. Research Note PNW-369, U.S. Forest Service.

McNeil, W., Ahnell, W., 1964. Success of pink salmon spawning relative to size of spawning bed materials. Spec. Scient. Rep. Fish. 469, U.S. Fish Wildi. Serv.
Peterson, N., Quinn, T., 1996. Persistence of egg pocket architecture in redds of chum salmon, Oncorhynchus keta. Environ. Biol. Fish. 46, 243-253.

Rykiel, E., 1996. Testing ecological model: the meaning of validation. Ecological modelling 90, 229-244.

Stuart, T., 1953. Spawning migration, reproduction and young stages of loch trout (Salmo trutta L.). Freshw. Salm. Fish. Res. 5, 1-39 Scottish Home Department.

van Winkle, W., Jager, H., Railsback, S., Holcomb, B., Studley, T., Baldrige, J., 1998. Individual-based model of sympatric populations of brown and rainbow trout for instream flow assessment: model description and calibration. Ecological Modelling 110, 175-207.

White, H., 1942. Atlantic salmon redds and artificial spawning beds. J. Fish. Res. Board Can. 6, 37-44. 